\title{
Geometrically Nonlinear Analysis of Structures Using Various Higher Order Solution Methods: A Comparative Analysis for Large Deformation
}

\author{
Ali Maghami ${ }^{1}$, Farzad Shahabian ${ }^{1}$ and Seyed Mahmoud Hosseini ${ }^{2, ~ *}$
}

\begin{abstract}
The suitability of six higher order root solvers is examined for solving the nonlinear equilibrium equations in large deformation analysis of structures. The applied methods have a better convergence rate than the quadratic Newton-Raphson method. These six methods do not require higher order derivatives to achieve a higher convergence rate. Six algorithms are developed to use the higher order methods in place of the NewtonRaphson method to solve the nonlinear equilibrium equations in geometrically nonlinear analysis of structures. The higher order methods are applied to both continuum and discrete problems (spherical shell and dome truss). The computational cost and the sensitivity of the higher order solution methods and the Newton-Raphson method with respect to the load increment size are comparatively investigated. The numerical results reveal that the higher order methods require a lower number of iterations that the Newton-Raphson method to converge. It is also shown that these methods are less sensitive to the variation of the load increment size. As it is indicated in numerical results, the average residual reduces in a lower number of iterations by the application of the higher order methods in the nonlinear analysis of structures.
\end{abstract}

Keywords: Geometrically nonlinear analysis, higher order methods, predictor-corrector algorithms, convergence rate, sensitivity to the increment size.

\section{Introduction}

For several decades nonlinear analysis of structures has been one of the most challenging issues in structural and mechanical engineering. In general, the nonlinear behavior of the structures is modeled as a system of algebraic nonlinear equations. In many practical modeling techniques, the computational methods such as the finite element method, Isogeometric method, boundary element method and the meshless method create a system of nonlinear equations [Han, Rajendran and Atluri (2005); Cai, Paik and Atluri (2009); Ghadiri Rad, Shahabian and Hosseini (2015a, 2015b); Nguyen-Thanh, Zhou, Zhuang et al. (2017)]. In most of structural systems analysis, the nonlinear response of the structure can present more applicable results than the linear one [Wang and Li (2015); Wang, Li, Wang

\footnotetext{
${ }^{1}$ Civil Engineering Department, Faculty of Engineering, Ferdowsi University of Mashhad, Mashhad, Iran.

${ }^{2}$ Industrial Engineering Department, Faculty of Engineering, Ferdowsi University of Mashhad, Mashhad, Iran. ${ }^{*}$ Corresponding Author: Seyed Mahmoud Hosseini. Email: sm_hosseini@um.ac.ir.
} 
et al. (2017)]. Especially in thin shells, consideration of large deformation is truly important, and several challenges regarding the complex geometry appears during the modeling process [Li, Nguyen-Thanh and Zhou (2018); Nguyen-Thanh, Li and Zhou (2018)]. It should be mentioned that several numerical techniques have been developed to improve the accuracy of the geometrically nonlinear analysis of the structures with complex geometry and materials [Schillinger, Düster and Rank (2012); Frikha, Wali, Hajlaoui et al. (2016); Hajlaoui, Triki, Frikha et al. (2017); Hajlaoui, Chebbi, Wali et al. (2019)]. During the analysis, the solution to the nonlinear equations is used to trace the equilibrium path and can be utilized to indicate the structure's response. Many attempts have been made to extend families of the predictor and corrector methods to solve the nonlinear equations [Liu and Atluri (2008); Liu, Yeih, Kuo et al. (2009); Liu and Atluri (2011)]. Different iterative methods have been developed as the alternative of Newton-Raphson method for solving nonlinear solid mechanics problems such as: modified Newton-Raphson [Crisfield (1979)], dynamic relaxation method [Brew and Brotton (1971); Alamatian (2012)], Jacobian free Newton Krylov methods [Hales, Novascone, Williamson et al. (2012)], preconditioned iterative techniques [Saffari, Maghami and Mansouri (2015)], optimization based method [Rezaiee-Pajand and Naserian (2018); Habibi and Bidmeshki (2019)], and a group method of data handling techniques [Nguyen, Lee, Nguyen-Xuan et al. (2019)]. Solving the equilibrium equations shows some disadvantages such as sensitivity to the increment size, numerous iterations and even failure in some cases [Carrera (1994); Ritto-Correa and Camotim (2008); Leon, Paulino, Pereira et al. (2011)]. Till date, the most dominant method to solve the nonlinear problems in structural mechanics is the classical Newton-Raphson method [Leon, Paulino, Pereira et al. (2011)]. The Newton-Raphson method is an iterative method which is based on Taylor series expansion. The Newton-Raphson method has been widely used as the main root solver to develop other techniques in many nonlinear practical problems such as pre-and post-buckling analysis [Pagani and Carrera (2017); Pagani, Carrera and Augello (2019)], damage detection procedures [Sotoudehnia, Shahabian and Aftabi-Sani (2019)] and power flow problems [Sereeter, Vuik and Witteveen (2019)]. It should be noted that the performance of Newton-Raphson method has a characteristic that makes it sensitive to initial prediction and step size that leads to the development of line search method to improve it [Kelley (2003)]. Several attempts have been done to improve the nonlinear solvers by improving the convergence rate in nonlinear structural analysis. One of the ways to increase the order of convergence is to employ higher order derivatives of the functions. The major weak point of these ways is the necessity of higher derivatives [Torkamani and Shieh (2011)]. On the other hand, computation of higher order derivatives requires lots of computational costs and it is impossible to compute higher order derivatives of the function in some cases.

The other accepted way to improve the convergence rate of an algorithm is to evaluate several functions at each iteration, which has been developed in the literature for both multivariate and scalar cases [Traub (1982); Petkovic, Neta, Petkovic et al. (2012)]. These procedures are named higher order multipoint methods. The most efficient schemes for solving nonlinear equations in both multivariate and scalar cases are multipoint iterative methods. To overcome the theoretical limits of one-point methods including computational efficiency and order of convergence, the multipoint methods are widely investigated recently [Petkovic, Neta, Petkovic et al. $(2012,2014)]$. In the last decade, because of the 
fast development of digital computers, a renewed interest in developing and utilizing these schemes has emerged. Recently, these methods have been applied to increase the ability of nonlinear solution techniques in different fields of engineering. Arroyo et al. [Arroyo, Cordero and Torregrosa (2011)] applied a higher order method to approximate the artificial satellite preliminary orbits. Six higher order methods are used instead of the NewtonRaphson method in $\mathrm{J} 2$ and Gurson plasticity constitutive model by Krian et al. [Krian, Li and Khandelwal (2015)]. The applicability of higher order nonlinear methods to solve the power flow equations is investigated by Derakhshandeh and Pourbagher [Derakhshandeh and Pourbagher (2016)]. The performance of higher order methods to solve the nonlinear equations in fracture analysis is tested by Kiran and Khandelwal [Kiran and Khandelwal (2018)]. More recently, Maghami et al. [Maghami, Shahabian and Hosseini (2018)], presented four modified techniques for the equilibrium path following of structures beyond the limit points based on the Multipoint methods.

The main novelty of this paper is to study the effect of convergence rate on the performance of geometrically nonlinear analysis in different fidelity levels. To achieve this purpose in the present study, six higher order methods with different convergence rate are employed to solve the nonlinear equilibrium equations arise in large deformation analysis of structures. The higher order methods consist of two third order, two fourth order and two fifth order convergence rate procedures. It worth noting that these higher order methods have been developed as iterative solvers. In this article, six algorithms are utilized that employs the higher order methods as the root solvers in an incremental-iterative manner for nonlinear analysis of the structures. As the number of increments controls the number of converged points and the fidelity level, the present study helps to understand the performance of higher order methods when deals with incremental process.

The main purposes of this study include, (i) to investigate the efficiency of six higher order iterative methods with different order of convergence rate in geometrically nonlinear analysis of structures, (ii) to evaluate the feasibility and computational cost of these procedures in practical nonlinear examples, (iii) to apply six step by step algorithms to use higher order method instead of Newton-Raphson in nonlinear analysis, (iv) to evaluate the sensitivity of higher order methods and the Newton-Raphson method with respect to the load increment size, (v) to investigate the convergence characteristics of different higher order methods and the average residual in nonlinear analysis of structure.

\section{Geometric nonlinearity and large deformation analysis of structures}

It is better to consider the geometric nonlinearities in all kinds of incremental analysis. To analyze the large deformation of structure it is necessary to apply the loads in an incremental manner and the geometric nonlinearity should be considered in the formulation. The consideration of geometric nonlinearities is well known in buckling, fabric structures, cable nets and so many other problems. The variation formulation of a body based on the principle of the virtual work is expressed as

$\delta W=\delta W_{\text {ext }}-\delta W_{\text {int }}=0$.

The internal work $\delta W_{\text {int }}$ can be described as 
$\delta W_{\text {int }}=\int_{V} \delta \mathbf{E}: \mathbf{S} d V$

where $\delta \mathbf{E}$ denotes the variation of strains, and $\mathbf{S}$ is the second Piola-Kirchhoff stress tensor. It worth mentioning that Eq. (2) can be written in details for any arbitrary solid element e.g., the internal virtual work for a shell element is integrated through the thickness of the shell as follows

$$
\int_{V} \delta \mathbf{E}: \mathbf{S} d V=\int_{A}(\mathbf{n}: \delta \boldsymbol{\varepsilon}+\mathbf{m}: \delta \mathbf{\kappa}) d A
$$

here, $\mathbf{n}=t \mathbf{C} \boldsymbol{\varepsilon}$ is the membrane stresses, and $\mathbf{m}=\left(t^{3} / 12\right) \mathbf{C} \boldsymbol{\kappa}$ denotes the bending stress with the material tensor C [Li, Nguyen-Thanh, Huang et al. (2020)]. The external work can be expressed as

$\delta W_{\mathrm{ext}}=\int_{A} \mathbf{b} \delta \mathbf{x} d A+\int_{\Gamma} \mathbf{h}_{\mathbf{0}} \delta \mathbf{x} d \Gamma$

where, $\mathbf{b}$ is the body force, and $\mathbf{h}_{\mathbf{0}}$ is the prescribed traction. The first derivative of the virtual work yields the unbalanced force vector:

$\mathbf{F}=\left(\frac{\partial W_{\text {ext }}}{\partial \mathbf{x}}-\frac{\partial W_{\text {int }}}{\partial \mathbf{x}}\right)=\mathbf{F}_{\text {ext }}-\mathbf{F}_{\text {int }}=\mathbf{0}$

here, $\mathbf{F}_{\text {ext }}$ is the external load vector, and $\mathbf{F}_{\text {int }}$ is the internal load vector.

In this paper, the higher order solution techniques are applied in nonlinear analysis of truss and shell examples. The stiffness matrix and the internal force vector are obtained based on load perturbation [Green, Knops and Laws (1968); Levy and Gal (2003); Gal and Levy (2006)] of the linear discrete equilibrium equations of an element in global coordinates. This formulation leads to the description of the stiffness matrix as the gradient of the nodal force vector. The equations of equilibrium for large deformation of structure can be written as

$\mathbf{N}^{\mathrm{T}} \mathbf{f}_{\text {int }}=\mathbf{F}_{\text {ext }}$

here, $\mathbf{f}_{\text {int }}$ is a vector containing the internal member forces resulting from stresses, $\mathbf{N}$ is an operator that describes the system geometric in equilibrium. Eq. (6) takes the below form under a perturbation

$\mathrm{d} \mathbf{N}^{\mathrm{T}} \mathbf{f}_{\text {int }}+\mathrm{N}^{\mathrm{T}} \mathrm{d} \mathbf{f}_{\text {int }}=\mathrm{d} \mathbf{F}_{\text {ext }}$.

The total displacement vector $(\mathbf{x})$ is used to represent the deformation of the structure. The nodal displacement variation vector $\boldsymbol{\delta}$ is the perturbation of the total displacement vector. By use of the chain rule of partial differentiation, the term $\mathrm{N}^{\mathrm{T}} \mathrm{d} \mathbf{f}_{\text {int }}$ in Eq. (7) indicates the variation of the member forces while the matrix $\mathbf{N}$ is fixed. In fact, this term of the equation is the linear elastic theory as follows

$\mathrm{N}^{\mathrm{T}} \mathrm{d} \mathbf{f}_{\text {int }} \rightarrow \mathbf{K}_{\mathrm{E}} \boldsymbol{\delta}$

here, $\mathbf{K}_{\mathrm{E}}$ is the elastic stiffness matrix. Whereas, the geometric stiffness matrix is obtained from the term $d \mathbf{N}^{\mathrm{T}} \mathbf{f}_{\text {int }}$ in Eq. (7) as follows 
$\mathrm{d}\left(\mathbf{N}^{\mathrm{T}} \mathbf{f}_{\text {int }}\right)_{\mathbf{f}_{\text {int fixed }}} \rightarrow \mathbf{K}_{\mathrm{G}} \boldsymbol{\delta}$

where, $\mathbf{K}_{\mathrm{G}}$ is the geometric stiffness matrix, and the following equation represents the relation between the displacements and the external forces

$\left(\mathbf{K}_{\mathrm{E}}+\mathbf{K}_{\mathrm{G}}\right) \boldsymbol{\delta}=\mathrm{d} \mathbf{F}_{\text {ext }}$

where this equation represents that a small change in the external forces leads to a variation in nodal displacement vector [Levy and Spillers (2013)]. The tangent stiffness matrix can be obtained as follows

$\mathbf{K}_{\mathrm{T}}=\mathbf{K}_{\mathrm{E}}+\mathbf{K}_{\mathrm{G}}$.

This matrix is obtainable by analytical methods only for some simple structures and it is constructed by numerical methods such as finite element and meshless for real problems. In this article, a two-node bar element is utilized to model the truss domes and the shell element is a combination of constant stress flat triangular element and the discrete Kirchhoff theory flat plate element [Levy and Spillers (2013)].

\subsection{The Geometric stiffness matrix of bar element}

The geometric stiffness matrix of a bar element is the gradient of ${ }^{e} \mathbf{N}^{\mathrm{T} e} \mathbf{f}_{\text {int }}$ [Levy and Spillers (2013)]. ${ }^{e} \mathbf{f}_{\text {int }}$ is the internal force of element $e$ and ${ }^{e} \mathbf{N}^{\mathrm{T}}$ is a vector that contains the geometric characteristics of the element in the following formula

${ }^{e} \mathbf{N}=\left\{\begin{array}{ll}{ }^{e} \mathbf{n} & -{ }^{e} \mathbf{n}\end{array}\right\}=\left\{\begin{array}{llllll}{ }^{e} n_{x} & { }^{e} n_{y} & { }^{e} n_{z} & -{ }^{e} n_{x} & -{ }^{e} n_{y} & -{ }^{e} n_{z}\end{array}\right\}$

where ${ }^{e} \mathbf{n}$ is a unit vector that is shown in Fig. 1 , and ${ }^{e} n_{x},{ }^{e} n_{x}$ and ${ }^{e} n_{z}$ are its componenets. The geometric stiffness matrix could be obtained as follows

$\mathbf{K}_{\mathrm{G}}=\nabla\left({ }^{e} \mathbf{N}^{\mathrm{T} e} \mathbf{f}_{\text {int }}\right)=\left[\begin{array}{cc}\mathbf{K}_{\mathrm{G}}^{\mathrm{sub}} & -\mathbf{K}_{\mathrm{G}}^{\mathrm{sub}} \\ -\mathbf{K}_{\mathrm{G}}^{\text {sub }} & \mathbf{K}_{\mathrm{G}}^{\text {sub }}\end{array}\right]$

$\mathbf{K}_{\mathrm{G}}^{\text {sub }}$ can be computed as

$\mathbf{K}_{\mathrm{G}}^{\mathrm{sub}}=\left(\frac{{ }^{e} \mathbf{f}_{\text {int }}}{{ }^{e} L}\right)\left(\mathbf{I}-{ }^{e} \mathbf{n}^{e} \mathbf{n}^{\mathrm{T}}\right)$ 


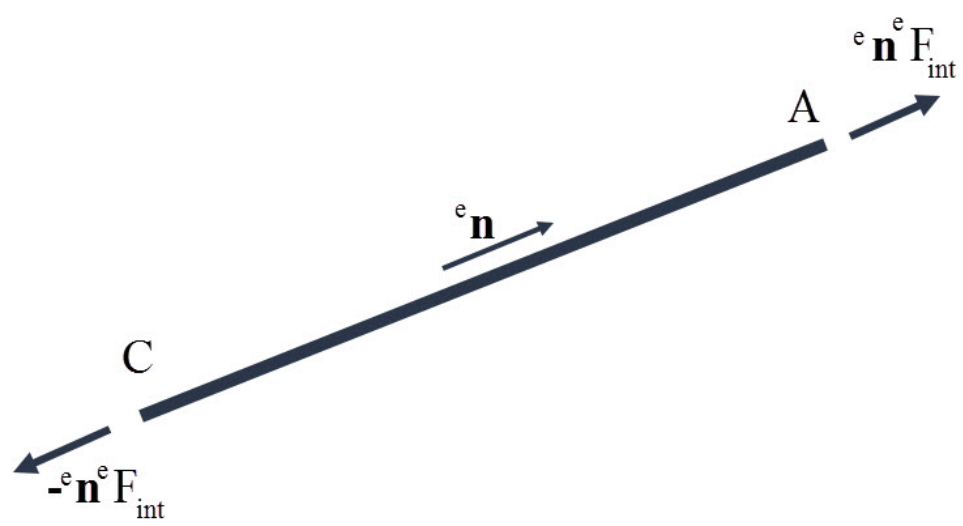

Figure 1: Bar element

\subsection{The Geometric stiffness matrix of shell element}

The geometric stiffness matrix of triangular shell element in local space can be split into three distinct matrices:

$$
\left[\mathbf{K}_{\mathrm{G}}\right]=\left[\mathbf{K}_{\mathrm{G}}\right]_{\mathrm{IP}}^{\mathrm{mem}}+\left[\mathbf{K}_{\mathrm{G}}\right]_{\mathrm{OP}}^{\text {shell }}+\left[\mathbf{K}_{\mathrm{G}}\right]_{\mathrm{IP}}^{\text {plate }}
$$

where the three terms on the right hand side of Eq. (15) is in-plane geometric stiffness matrix of the triangular membrane element, out-of-plane geometric stiffness matrix of triangular shell element and in-plane geometric stiffness matrix of triangular plate element respectively. It is important to note that the tangential stiffness also includes the linear elastic stiffness that is formed as follows

$$
\left[\mathbf{K}_{\mathrm{E}}\right]=\left[\mathbf{K}_{\mathrm{E}}\right]^{\mathrm{mem}}+\left[\mathbf{K}_{\mathrm{E}}\right]^{\text {plate }}
$$

where first and second terms on the right hand side of Eq. (16) are the linear elastic stiffness matrix of plane stress triangular element (Fig. 2) and linear elastic stiffness matrix of triangular plate element (Fig. 3). Therefore, the final geometrically nonlinear triangular shell element has eighteen degrees of freedom including three displacements and three rotations at each node. A detail description on the stiffness matrices is explained by Levy et al. [Levy and Spillers (2013)].

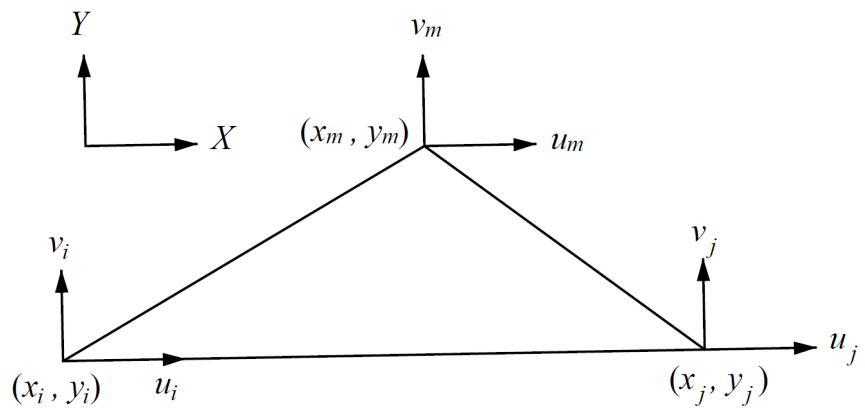

Figure 2: Triangular membrane element 


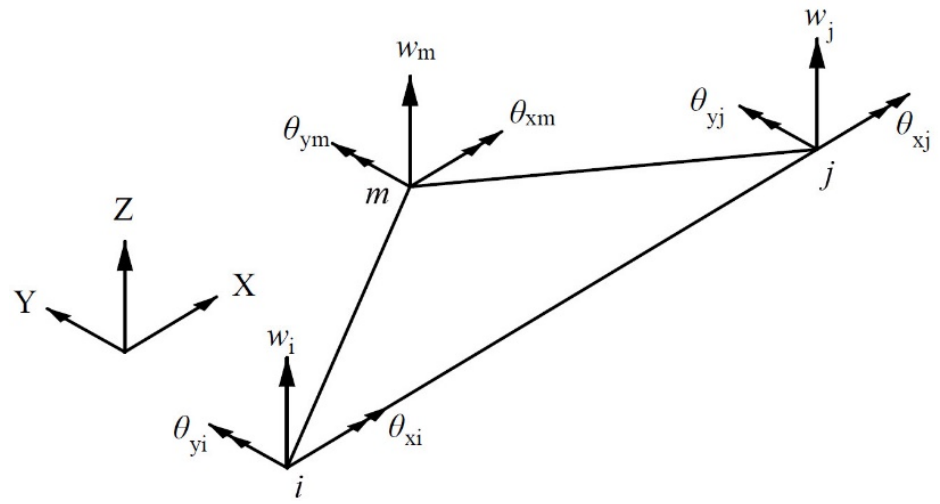

Figure 3: Triangular plate element

\section{The Newton-Raphson and higher order methods for nonlinear analysis}

Let $\mathbf{F}$ be a real function. If $\mathbf{F}(\boldsymbol{\alpha})=\mathbf{0}$ then $\boldsymbol{\alpha}$ is called a root of the equation

$\mathbf{F}(\mathbf{x})=\mathbf{0}$

As it is obvious, the roots of Eq. (5) can be obtained analytically only for some special cases. The mathematical formulation of the Newton-Raphson (NR) method to find the roots of Eq. (5) in an iterative manner is

$\mathbf{x}_{n+1}=\mathbf{x}_{n}-\mathbf{F}^{\prime}\left(\mathbf{x}_{n}\right)^{-1} \mathbf{F}\left(\mathbf{x}_{n}\right)$

where, $\mathbf{F}^{\prime}\left(\mathbf{x}_{n}\right) \in \mathbf{R}^{n \times n}$ is a nonsingular Jacobian matrix which is given as

$\mathbf{F}^{\prime}\left(\mathbf{x}_{n}\right)=\left[\begin{array}{ccc}\frac{\partial F_{1}}{\partial x_{1}} & \cdots & \frac{\partial F_{1}}{\partial x_{m}} \\ \vdots & \ddots & \vdots \\ \frac{\partial F_{m}}{\partial x_{1}} & \cdots & \frac{\partial F_{m}}{\partial x_{m}}\end{array}\right]_{x=x_{n}}$

The higher order multipoint methods are effective numerical techniques that can be applied in solving any nonlinear equation [Petkovic, Neta, Petkovic et al. (2012)]. Six higher order methods are addressed here. It should be noted that these higher order methods are applied instead of the Newton-Raphson method to solve the nonlinear equilibrium equations of structures in this paper. These higher order methods do not require higher order derivatives to achieve a higher convergence rate.

\subsection{Homeier method (HM)}

A two-steps cubically convergent method was developed by Homeier [Homeier (2004)] for the multivariate case, which can be given as

$\mathbf{y}_{n}=\mathbf{x}_{n}-\frac{1}{2} \mathbf{F}^{\prime}\left(\mathbf{x}_{n}\right)^{-1} \mathbf{F}\left(\mathbf{x}_{n}\right)$ 
$\mathbf{x}_{n+1}=\mathbf{x}_{n}-\mathbf{F}^{\prime}\left(\mathbf{y}_{n}\right)^{-1} \mathbf{F}\left(\mathbf{x}_{n}\right)$

This method was earlier presented as a modification of the Newton-Raphson method for finding the root of a univariate function [Homeier (2003)]. In each iteration, the procedure needs one evaluation of the function vector and the Jacobian has to be evaluated twice for solving two linear systems with the Jacobian as the coefficient matrix.

\subsection{Weerakoon and Fernando method (WFM)}

The two-step method for solving the nonlinear equations is as follows

$\mathbf{y}_{n}=\mathbf{x}_{n}-\frac{1}{2} \mathbf{F}^{\prime}\left(\mathbf{x}_{n}\right)^{-1} \mathbf{F}\left(\mathbf{x}_{n}\right)$

$\mathbf{x}_{n+1}=\mathbf{x}_{n}-2\left[\mathbf{F}^{\prime}\left(\mathbf{y}_{n}\right)+\mathbf{F}^{\prime}\left(\mathbf{x}_{n}\right)\right]^{-1} \mathbf{F}\left(\mathbf{x}_{n}\right)$.

This iterative third order method is a special case of Traub's techniques [Traub (1982)]. In this iterative method, the Jacobian matrix is evaluated two times in each iteration.

\subsection{Jarrat method (JM)}

The fourth order Jarratt's method [Jarrat (1966)] is given as

$$
\begin{aligned}
& \mathbf{y}_{n}=\mathbf{x}_{n}-\frac{2}{3} \mathbf{F}^{\prime}\left(\mathbf{x}_{n}\right)^{-1} \mathbf{F}\left(\mathbf{x}_{n}\right) \\
& \mathbf{x}_{n+1}=\mathbf{x}_{n}-\left[3 \mathbf{F}^{\prime}\left(\mathbf{y}_{n}\right)-\mathbf{F}^{\prime}\left(\mathbf{x}_{n}\right)\right]^{-1}\left[3 \mathbf{F}^{\prime}\left(\mathbf{y}_{n}\right)+\mathbf{F}^{\prime}\left(\mathbf{x}_{n}\right)\right] \mathbf{F}^{\prime}\left(\mathbf{x}_{n}\right)^{-1} \mathbf{F}\left(\mathbf{x}_{n}\right) .
\end{aligned}
$$

At first, this fourth order procedure was proposed by Jarrat (1966) for the univariate case. Later, this method is applied in multivariate cases in many studies [Sharma and Sharma (2010); Sharma and Gupta (2014)]. This two-steps procedure requires the evaluations of one function, two first derivatives and two matrix inversions.

\subsection{Darvishi and Barati method (DBM)}

The fourth order Darvishi and Barati method [Darvishi and Barati (2007a)] is defined as

$\mathbf{y}_{n}=\mathbf{x}_{n}-\mathbf{F}^{\prime}\left(\mathbf{x}_{n}\right)^{-1} \mathbf{F}\left(\mathbf{x}_{n}\right)$,

$\mathbf{z}_{n}=\mathbf{x}_{n}-\mathbf{F}^{\prime}\left(\mathbf{x}_{n}\right)^{-1}\left(\mathbf{F}\left(\mathbf{x}_{n}\right)+\mathbf{F}\left(\mathbf{y}_{n}\right)\right)$,

$\mathbf{x}_{n+1}=\mathbf{x}_{n}-\left[\frac{1}{6} \mathbf{F}^{\prime}\left(\mathbf{x}_{n}\right)+\frac{2}{3} \mathbf{F}^{\prime}\left(\frac{\mathbf{x}_{n}+\mathbf{z}_{n}}{2}\right)+\frac{1}{6} \mathbf{F}^{\prime}\left(\mathbf{z}_{n}\right)\right]^{-1} \mathbf{F}\left(\mathbf{x}_{n}\right)$.

This method is a modification of a third order method presented by Darvishi et al. [Darvishi and Barati (2007b)]. DBM requires the evaluations of two functions, three first derivatives and two matrix inversions.

\subsection{Cordero and Torregrosa method (CTM)}

The fifth order method presented by Cordero et al. [Cordero and Torregrosa (2007)] is given as 
$\mathbf{y}_{n}=\mathbf{x}_{n}-\mathbf{F}^{\prime}\left(\mathbf{x}_{n}\right)^{-1} \mathbf{F}\left(\mathbf{x}_{n}\right)$,

$\mathbf{z}_{n}=\mathbf{x}_{n}-2\left[\mathbf{F}^{\prime}\left(\mathbf{x}_{n}\right)+\mathbf{F}^{\prime}\left(\mathbf{y}_{n}\right)\right]^{-1} \mathbf{F}\left(\mathbf{x}_{n}\right)$,

$\mathbf{x}_{n+1}=\mathbf{z}_{n}-\mathbf{F}^{\prime}\left(\mathbf{y}_{n}\right)^{-1} \mathbf{F}\left(\mathbf{z}_{n}\right)$.

In this three-step method two function evaluations, two Jacobean matrix evaluations and three matrix inversions are required.

\subsection{Sharma and Gupta method (SGM)}

This method is a fifth order convergence technique that is proposed by Sharma et al. [Sharma and Gupta (2014)] as

$\mathbf{y}_{n}=\mathbf{x}_{n}-\frac{1}{2} \mathbf{F}^{\prime}\left(\mathbf{x}_{n}\right)^{-1} \mathbf{F}\left(\mathbf{x}_{n}\right)$

$\mathbf{z}_{n}=\mathbf{x}_{n}-\mathbf{F}^{\prime}\left(\mathbf{y}_{n}\right)^{-1} \mathbf{F}\left(\mathbf{x}_{n}\right)$,

$\mathbf{x}_{n+1}=\mathbf{z}_{n}-\left[2 \mathbf{F}^{\prime}\left(\mathbf{y}_{n}\right)^{-1}-\mathbf{F}^{\prime}\left(\mathbf{x}_{n}\right)^{-1}\right] \mathbf{F}\left(\mathbf{z}_{n}\right)$.

In each iteration of SGM, two function evaluation and two matrix inventions are needed.

4 The implementation of the higher order methods in nonlinear analysis of structures

The nonlinear analysis of structures is an incremental-iterative manner and the solution algorithms are called predictor-corrector techniques. In this section, the higher order methods are presented in an incremental-iterative framework. Three step by step algorithms are presented to apply the higher order methods to solve the nonlinear equilibrium equations. The HM, WFM and JM techniques have two steps in each iteration. Therefore, the corresponding algorithms have many steps in common. The first three methods are represented in Algorithm 1. Algorithm 2 is utilized to represent DBM technique. The CTM and SGM are utilized to develop Algorithm 3. 
Algorithm 1: The implementation of HM, WFM and JM.

Initialization

For $j=1$ : $n$ inc

$\left(\mathbf{F}_{\text {ext }}\right)^{j} \leftarrow\left(\mathbf{F}_{\text {ext }}\right)^{j-1}+\Delta \mathbf{F}_{\text {ext }}$

While $\operatorname{Res}_{i}^{j} \leq$ tol for $i=1$ : maxit

Calculate $\left(\mathbf{F}_{\text {int }}\right)_{i}^{j}$ and $\left(\mathbf{K}_{\mathrm{Tx}}\right)_{i}^{j}$ according to $\mathbf{x}_{i}^{j}$

Compute the unbalanced force $(\mathbf{F})_{i}^{j}=\left(\mathbf{F}_{\text {ext }}\right)_{i}^{j}-\left(\mathbf{F}_{\text {int }}\right)_{i}^{j}$

\begin{tabular}{cl}
\hline HM and WFM & $\left(\boldsymbol{\delta}_{y}\right)_{i}^{j}=\frac{1}{2}\left[\left(\mathbf{K}_{\mathrm{Tx}}\right)_{i}^{j}\right]^{-1}(\mathbf{F})_{i}^{j}$ \\
\hline $\mathrm{JM}$ & $\left(\boldsymbol{\delta}_{y}\right)_{i}^{j}=\frac{2}{3}\left[\left(\mathbf{K}_{\mathrm{Tx}}\right)_{i}^{j}\right]^{-1}(\mathbf{F})_{i}^{j}$
\end{tabular}

Update the global nodal displacement $\mathbf{y}_{i}^{j} \leftarrow \mathbf{x}_{i}^{j}+\left(\boldsymbol{\delta}_{y}\right)_{i}^{j}$

Calculate $\left(\mathbf{K}_{\mathrm{Ty}}\right)_{i}^{j}$ according to $\mathbf{y}_{i}^{j}$

\begin{tabular}{cc}
\hline $\mathrm{HM}$ & $\left(\boldsymbol{\delta}_{x}\right)_{i}^{j}=\left[\left(\mathbf{K}_{\mathrm{Ty}}\right)_{i}^{j}\right]^{-1}(\mathbf{F})_{i}^{j}$ \\
\hline $\mathrm{WFM}$ & $\left(\boldsymbol{\delta}_{x}\right)_{i}^{j}=2\left[\left(\mathbf{K}_{\mathrm{Tx}}\right)_{i}^{j}+\left(\mathbf{K}_{\mathrm{Ty}}\right)_{i}^{j}\right]^{-1}(\mathbf{F})_{i}^{j}$ \\
\hline $\mathrm{JM}$ & $\left(\boldsymbol{\delta}_{x}\right)_{i}^{j}=\left[3\left(\mathbf{K}_{\mathrm{Tx}}\right)_{i}^{j}-\left(\mathbf{K}_{\mathrm{Ty}}\right)_{i}^{j}\right]^{-1}\left[3\left(\mathbf{K}_{\mathrm{Tx}}\right)_{i}^{j}+\left(\mathbf{K}_{\mathrm{Ty}}\right)_{i}^{j}\right]\left[\left(\mathbf{K}_{\mathrm{Tx}}\right)_{i}^{j}\right]^{-1}(\mathbf{F})_{i}^{j}$ \\
\hline
\end{tabular}

Update the global nodal displacement $\mathbf{x}_{i+1}^{j} \leftarrow \mathbf{x}_{i}^{j}+\left(\boldsymbol{\delta}_{x}\right)_{i}^{j}$

\section{End}

Save the converged load and displacement

End 
Algorithm 2: The implementation of DBM.

Initialization

For $j=1$ : ninc

$\left(\mathbf{F}_{\text {ext }}\right)^{j} \leftarrow\left(\mathbf{F}_{\text {ext }}\right)^{j-1}+\Delta \mathbf{F}_{\text {ext }}$

While $\operatorname{Res}_{i}^{j} \leq$ tol for $i=1$ : maxit

Calculate $\left(\mathbf{F}_{\text {int }}\right)_{i}^{j}$ and $\left(\mathbf{K}_{\mathrm{Tx}}\right)_{i}^{j}$ according to $\mathbf{x}_{i}^{j}$

Compute the unbalanced force $(\mathbf{F})_{i}^{j}=\left(\mathbf{F}_{\text {ext }}\right)_{i}^{j}-\left(\mathbf{F}_{\text {int }}\right)_{i}^{j}$

$\left(\boldsymbol{\delta}_{y}\right)_{i}^{j}=\left[\left(\mathbf{K}_{\mathrm{Tx}}\right)_{i}^{j}\right]^{-1}(\mathbf{F})_{i}^{j}$

Update the global nodal displacement $\mathbf{y}_{i}^{j} \leftarrow \mathbf{x}_{i}^{j}+\left(\boldsymbol{\delta}_{y}\right)_{i}^{j}$

Calculate $\left(\mathbf{F}_{\text {int } y}\right)_{i}^{j}$ according to $\mathbf{y}_{i}^{j}$

Compute the unbalanced force $\left(\mathbf{F}_{y}\right)_{i}^{j}=\left(\mathbf{F}_{\text {ext }}\right)_{i}^{j}-\left(\mathbf{F}_{\text {int } y}\right)_{i}^{j}$

$\left(\boldsymbol{\delta}_{z}\right)_{i}^{j}=\left[\left(\mathbf{K}_{\mathrm{Tx}}\right)_{i}^{j}\right]^{-1}\left[(\mathbf{F})_{i}^{j}+\left(\mathbf{F}_{y}\right)_{i}^{j}\right]$

Update the global nodal displacement $\mathbf{z}_{i}^{j} \leftarrow \mathbf{x}_{i}^{j}+\left(\boldsymbol{\delta}_{z}\right)_{i}^{j}$

Calculate $\left(\mathbf{K}_{\mathrm{Tz}}\right)_{i}^{j}$ according to $\mathbf{z}_{i}^{j}$

Calculate $\left(\mathbf{K}_{\mathrm{Txz}}\right)_{i}^{j}$ according to $\left(\mathbf{x}_{i}^{j}+\mathbf{z}_{i}^{j}\right) / 2$

$\left(\boldsymbol{\delta}_{x}\right)_{i}^{j}=\left[\frac{1}{6}\left(\mathbf{K}_{\mathrm{Tx}}\right)_{i}^{j}+\frac{2}{3}\left(\mathbf{K}_{\mathrm{Txz}}\right)_{i}^{j}+\frac{1}{6}\left(\mathbf{K}_{\mathrm{Tz}}\right)_{i}^{j}\right]^{-1}(\mathbf{F})_{i}^{j}$

Update the global nodal displacement $\mathbf{x}_{i+1}^{j} \leftarrow \mathbf{x}_{i}^{j}+\left(\boldsymbol{\delta}_{x}\right)_{i}^{j}$

\section{End}

Save the converged load and displacement

End 
Algorithm 3: Implementation of CTM and SGM

Initialization

For $j=1$ : $n$ inc

$\left(\mathbf{F}_{\text {ext }}\right)^{j} \leftarrow\left(\mathbf{F}_{\text {ext }}\right)^{j-1}+\Delta \mathbf{F}_{\text {ext }}$

While $\operatorname{Res}_{i}^{j} \leq$ tol for $i=1$ : maxit

Calculate $\left(\mathbf{F}_{\text {int } y}\right)_{i}^{j}$ and $\left(\mathbf{K}_{\mathrm{Tx}}\right)_{i}^{j}$ according to $\mathbf{x}_{i}^{j}$

Compute the unbalanced force $(\mathbf{F})_{i}^{j}=\left(\mathbf{F}_{\text {ext }}\right)_{i}^{j}-\left(\mathbf{F}_{\text {int }}\right)_{i}^{j}$

\begin{tabular}{ll}
\hline CTM & $\left(\boldsymbol{\delta}_{y}\right)_{i}^{j}=\left[\left(\mathbf{K}_{\mathrm{Tx}}\right)_{i}^{j}\right]^{-1}(\mathbf{F})_{i}^{j}$ \\
\hline $\mathrm{SGM}$ & $\left(\boldsymbol{\delta}_{y}\right)_{i}^{j}=\frac{1}{2}\left[\left(\mathbf{K}_{\mathrm{Tx}}\right)_{i}^{j}\right]^{-1}(\mathbf{F})_{i}^{j}$ \\
\hline
\end{tabular}

Update the global nodal displacement $\mathbf{y}_{i}^{j} \leftarrow \mathbf{x}_{i}^{j}+\left(\boldsymbol{\delta}_{y}\right)_{i}^{j}$

Calculate $\left(\mathbf{K}_{\mathrm{Ty}}\right)_{i}^{j}$ according to $\mathbf{y}_{i}^{j}$

\begin{tabular}{cc}
\hline CTM & $\left(\boldsymbol{\delta}_{z}\right)_{i}^{j}=2\left[\left(\mathbf{K}_{\mathrm{Tx}}\right)_{i}^{j}+\left(\mathbf{K}_{\mathrm{Ty}}\right)_{i}^{j}\right]^{-1}(\mathbf{F})_{i}^{j}$ \\
\hline $\mathrm{SGM}$ & $\left(\boldsymbol{\delta}_{z}\right)_{i}^{j}=\left[\left(\mathbf{K}_{\mathrm{Ty}}\right)_{i}^{j}\right]^{-1}(\mathbf{F})_{i}^{j}$
\end{tabular}

Update the global nodal displacement $\mathbf{z}_{i}^{j} \leftarrow \mathbf{x}_{i}^{j}+\left(\boldsymbol{\delta}_{z}\right)_{i}^{j}$

Calculate $\left(\mathbf{F}_{\text {int } z}\right)_{i}^{j}$ and $\left(\mathbf{F}_{z}\right)_{i}^{j}=\left(\mathbf{F}_{\text {ext }}\right)_{i}^{j}-\left(\mathbf{F}_{\text {int } z}\right)_{i}^{j}$ according to $\mathbf{z}_{i}^{j}$

\begin{tabular}{cc}
\hline CTM & $\left(\boldsymbol{\delta}_{x}\right)_{i}^{j}=\left[\left(\mathbf{K}_{\mathrm{Ty}}\right)_{i}^{j}\right]^{-1}\left(\mathbf{F}_{z}\right)_{i}^{j}$ \\
\hline $\mathrm{SGM}$ & $\left(\boldsymbol{\delta}_{x}\right)_{i}^{j}=\left[2\left[\left(\mathbf{K}_{\mathrm{Ty}}\right)_{i}^{j}\right]^{-1}-\left[\left(\mathbf{K}_{\mathrm{Tx}}\right)_{i}^{j}\right]^{-1}\right]\left(\mathbf{F}_{z}\right)_{i}^{j}$ \\
\hline
\end{tabular}

Update the global nodal displacement $\mathbf{x}_{i+1}^{j} \leftarrow \mathbf{z}_{i}^{j}+\left(\boldsymbol{\delta}_{x}\right)_{i}^{j}$

\section{End}

Save the converged load and displacement

End 
In the developed Algorithms, the upper index is used to show the number of increment $(j)$, the lower index shows the number of iteration $(i)$, and maxit is the maximum number of iterations. Also, ninc denotes the maximum number of load increments. In fact, the maximum number of load increments specifies the increment size as follows

$\Delta \mathbf{F}_{\mathrm{ext}}=\frac{\mathbf{F}_{\mathrm{ext}}}{\operatorname{ninc}}$

where, $\mathbf{F}_{\text {ext }}$ is the total external load vector that is applied on the structures in an incremental manner by equal increment size of $\Delta \mathbf{F}_{\text {ext }}$. It should be emphasize that the number of converged points on the equilibrium is equal to ninc. Therefore, the level of fidelity of the obtained equilibrium path is dependent to the maximum number of load increments. As it was mentioned before, the main goal of this paper is to study the performance of different higher order methods in different fidelity levels. As a consequence, the numerical analysis is done in different ninc. In the presented algorithms, the convergence criterion is defined as

$\operatorname{Res}_{i}^{j}=\frac{\left\|(\mathbf{F})_{i}^{j}\right\|}{\left\|\mathbf{F}_{\text {ext }}\right\|} \leq$ tol

here, $\operatorname{Res}_{i}^{j}$ is the residual and $t o l$ is the tolerance [De Borst, Crisfield, Remmers et al. (2012)]. To show the characteristics of different methods for solving the nonlinear equilibrium equations, the average residual in each analysis case is computed. The average residual is the mean of the residual in different loading steps at a certain iteration which is computed as follows

$$
A R^{j}=\frac{\sum_{i=1}^{n i n c} \operatorname{Res}_{i}^{j}}{\operatorname{ninc}}
$$

\section{Numerical examples and discussions}

The efficiency of the higher order methods for nonlinear analysis of structures is comparatively explored in this section. Two examples were solved for numerical verification and evaluation of the presented algorithms. A computer program based on the presented procedures is developed in MATLAB. In order to evaluate the number of iterations, the nonlinear equilibrium path for each example is followed in an incrementaliterative manner. Each example is solved by 95 different load increment size and the sensitivity of the algorithms with respect to the change in the load increment size is evaluated. The number of load increments varies from 5 to 100 . The tolerance $(t o l)$ is set to $1 e-10$. The maximum number of iteration is equal to 20 in all the analysis. The definitions of the terms utilized in numerical results are presented in Tab. 1. 
Table 1: The abbreviations and their definitions in numerical results

\begin{tabular}{cc}
\hline Term & Definition \\
\hline NR & Newton-Raphson \\
HM & Homeier Method \\
WFM & Weerakoon and Fernando Method \\
JM & Jarrat Method \\
DBM & Darvishi and Barati Method \\
CTM & Cordero and Torregrosa method \\
SGM & Sharma and Gupta Method \\
NIT & Number of Iterations \\
NUE & Number of Unbalanced Force Evaluations \\
NSE & Number of Stiffness Matrix Evaluations \\
NMI & Number of Matrix Inversions \\
RPR\% & Relative Percentage Reduction \% \\
AR & Average Residual \\
ninc & Maximum Number of Increments \\
\hline
\end{tabular}

\subsection{Schewdeler's dome}

The first example shown in Fig. 1, is a truss dome with 264 elements and 97 nodes. The axial stiffness of all elements is $E A=6.4 e+5 \mathrm{kN}$ and the structure has 291 DOFs. The structure's hinged supports are shown as black nodes in Fig. 4. A downward load $P=30$ $\mathrm{kN}$ is applied at the apex of this dome truss. This structure has been used to evaluate several numerical methods [Greco, Gesualdo, Venturini et al. (2006); Saffari, Maghami and Mansouri (2015)].

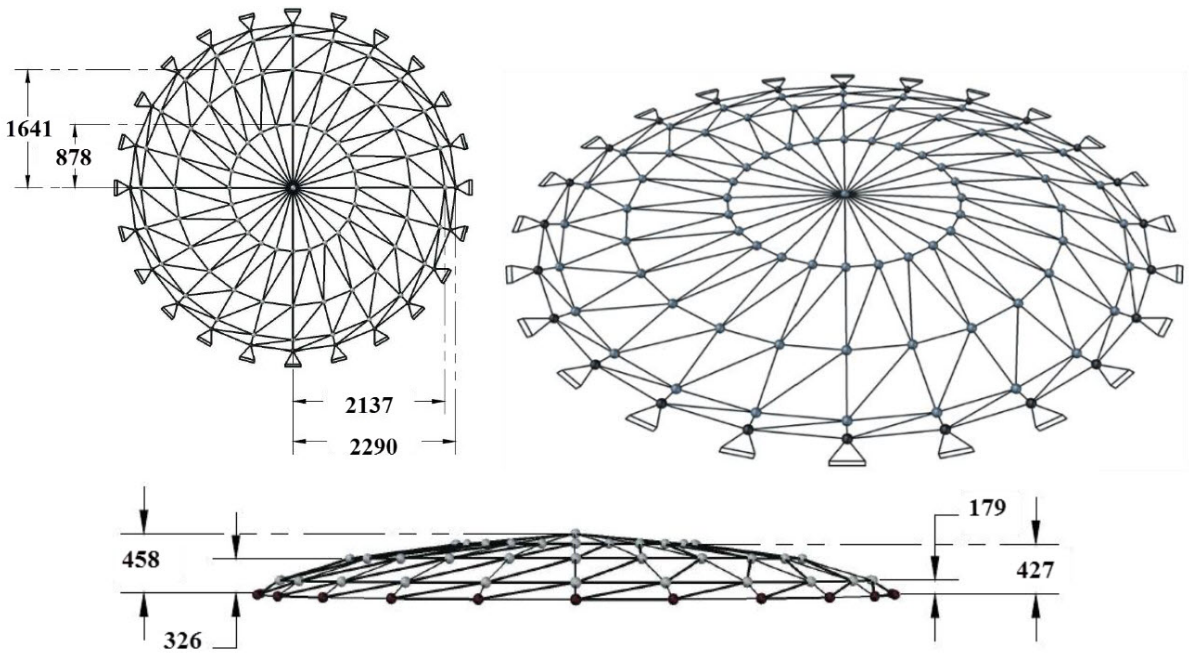

Figure 4: Schewdeler's dome truss (dimensions are given in $\mathrm{cm}$ ) 
The Schewdeler's dome truss is analyzed by the represented algorithms and the NewtonRaphson method. The load-displacement curve of the apex node is depicted in Fig. 5. As can be seen in this figure, the load-deformation curves that are obtained from the higher order methods and the Newton-Raphson are nearly the same. The contour plots of stress in the deformed configuration is depicted in Fig. 6. Fig. 7 shows the contour plots of displacements in the last converged point on the equilibrium path.

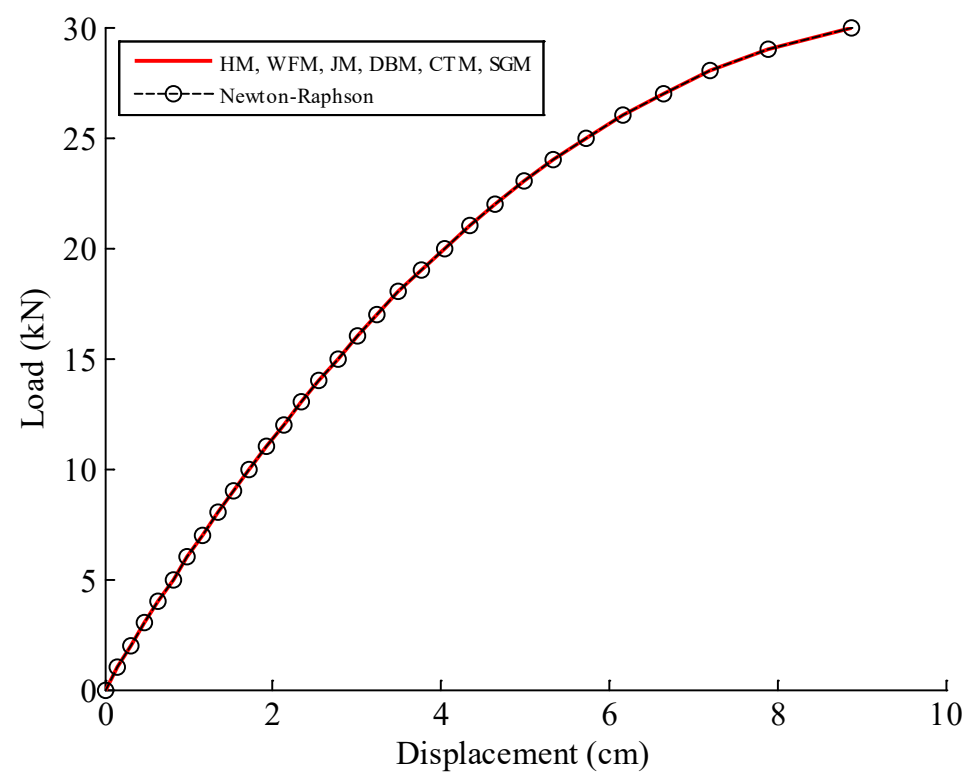

Figure 5: The nonlinear load-displacement curve of Schewdeler's dome truss
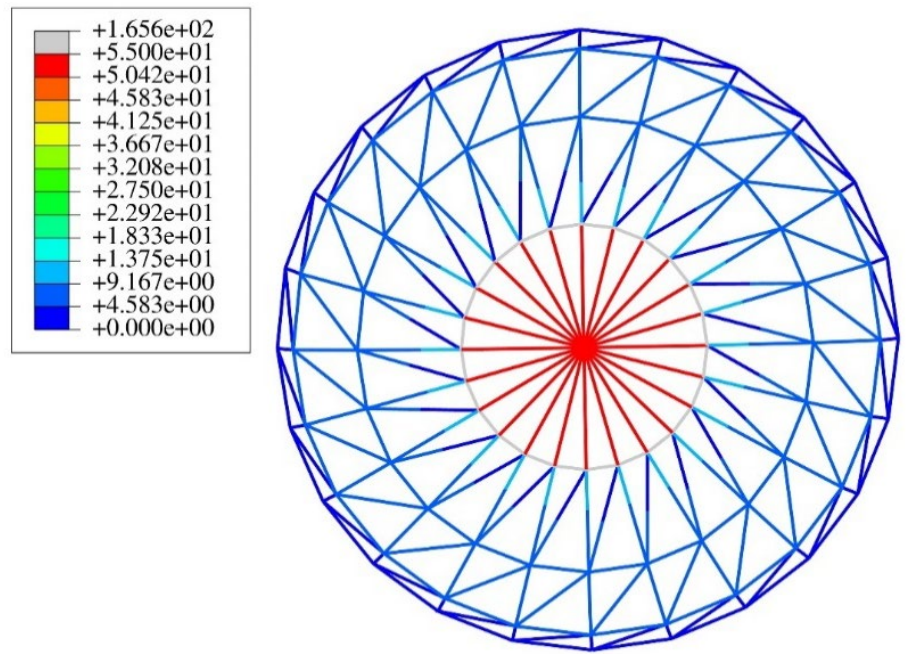

Figure 6: The contour plots of stress in Schewdeler's dome truss after deformation 

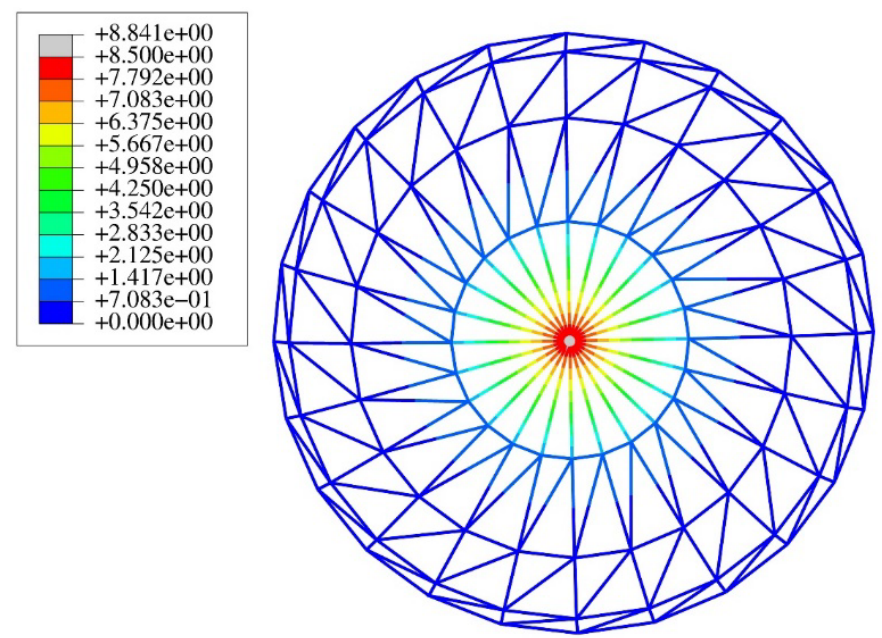

Figure 7: The contour plots of displacements in Schewdeler's dome truss after deformation

To investigate the sensitivity of the higher order root solvers with respect to the variation of increment size, the nonlinear analysis of Schewdeler's dome truss is conducted by various ninc. The number of load increments varies from 5 to 100 . The results related to the analysis of the Schewdeler's dome truss in five different analysis cases $(5,25,50,75$ and 100) are provided in Tab. 2. As can be seen in this table, all the higher order methods require a lower number of iterations to converge in all the loading cases. As it was mentioned earlier, the HM and WFM have the same order of convergence, JM and DBM are fourth order methods and CTM and SGM are the methods with fifth order of convergence. Based on Tab. 2, every two methods that have the same converge rate (e.g., HM and WFM) show the similar performance during the analysis and the required number of iterations in the nonlinear analysis of the truss dome is nearly the same. In this table, among the higher order methods, CTM has the most value of RPR in all the analysis cases and HM and WFM require the least computational cost. It is reflected in Tab. 2 that the raise in maximum number of load increment (increase in fidelity level) leads to an increase in the required number of iterations to show the convergence.

Fig. 8 shows the average residual in each iteration. This figure can be considered as the main result that shows the cause of the difference occurred during the analysis by different higher order methods. The characteristics and the accelerated convergence rate of the presented algorithms in three different load increments are shown in this figure. As can be seen in Fig. 8, the higher order methods accelerate the convergence rate and the NewtonRaphson method requires more iterations to reduce the average residual when it is compared to the higher order methods. It is clear in Fig. 8, which the cubic convergence methods (HM and WFM) have the same behavior and the required number of iterations by these methods is more than the other higher order techniques to reduce the average residual. As indicated in Fig. 8, the fifth order methods follow shorter paths to achieve the convergence. In fact, this figure shows that different methods follow the various paths for the convergence. These convergence paths are the reason that these methods have different 
characteristics. It is obvious that all the higher order methods have a better convergence characteristics than the Newton-Raphson method.

Table 2: comparison of different methods in various number of load increments for analysis of Schewdeler's dome truss

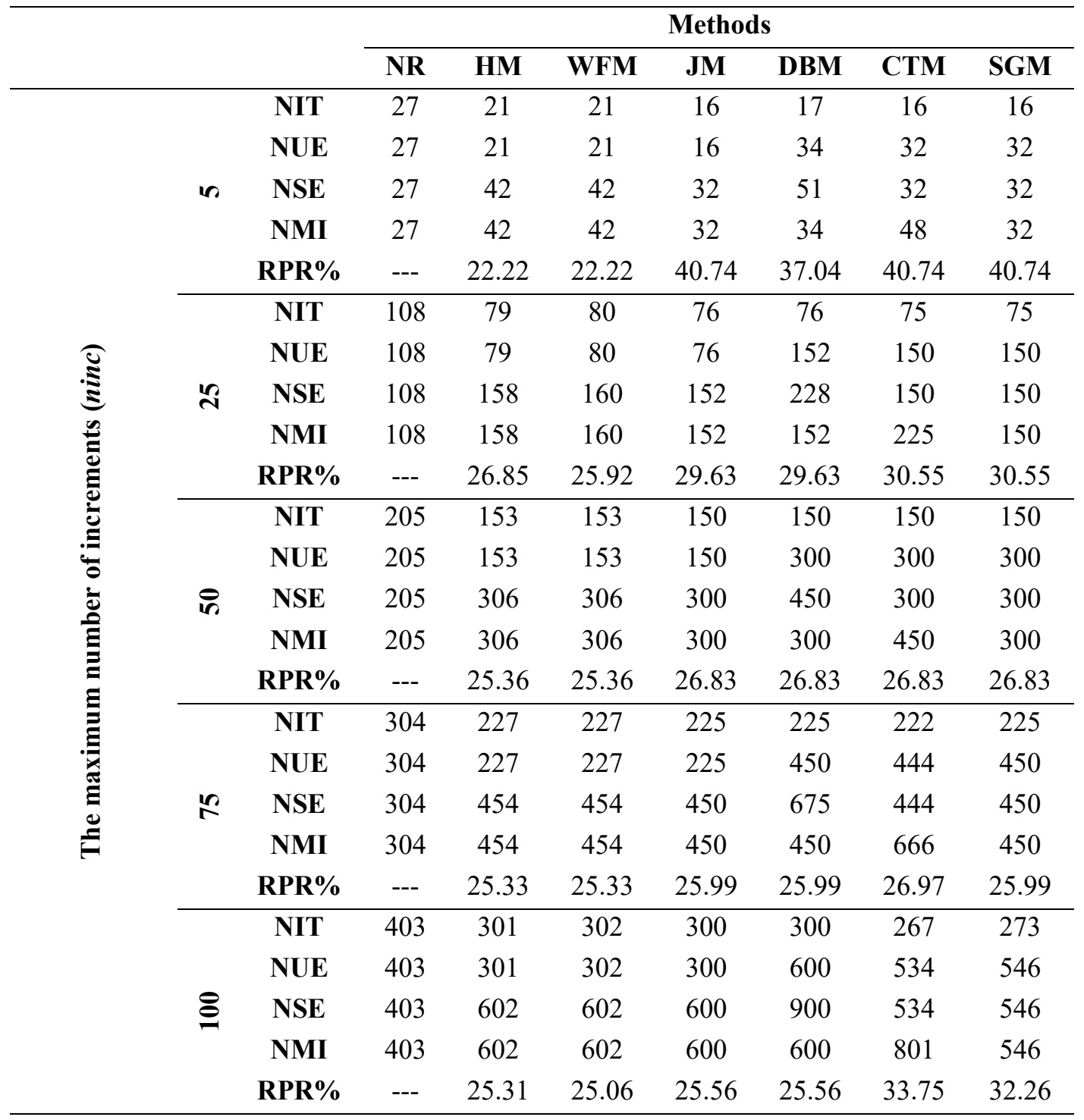



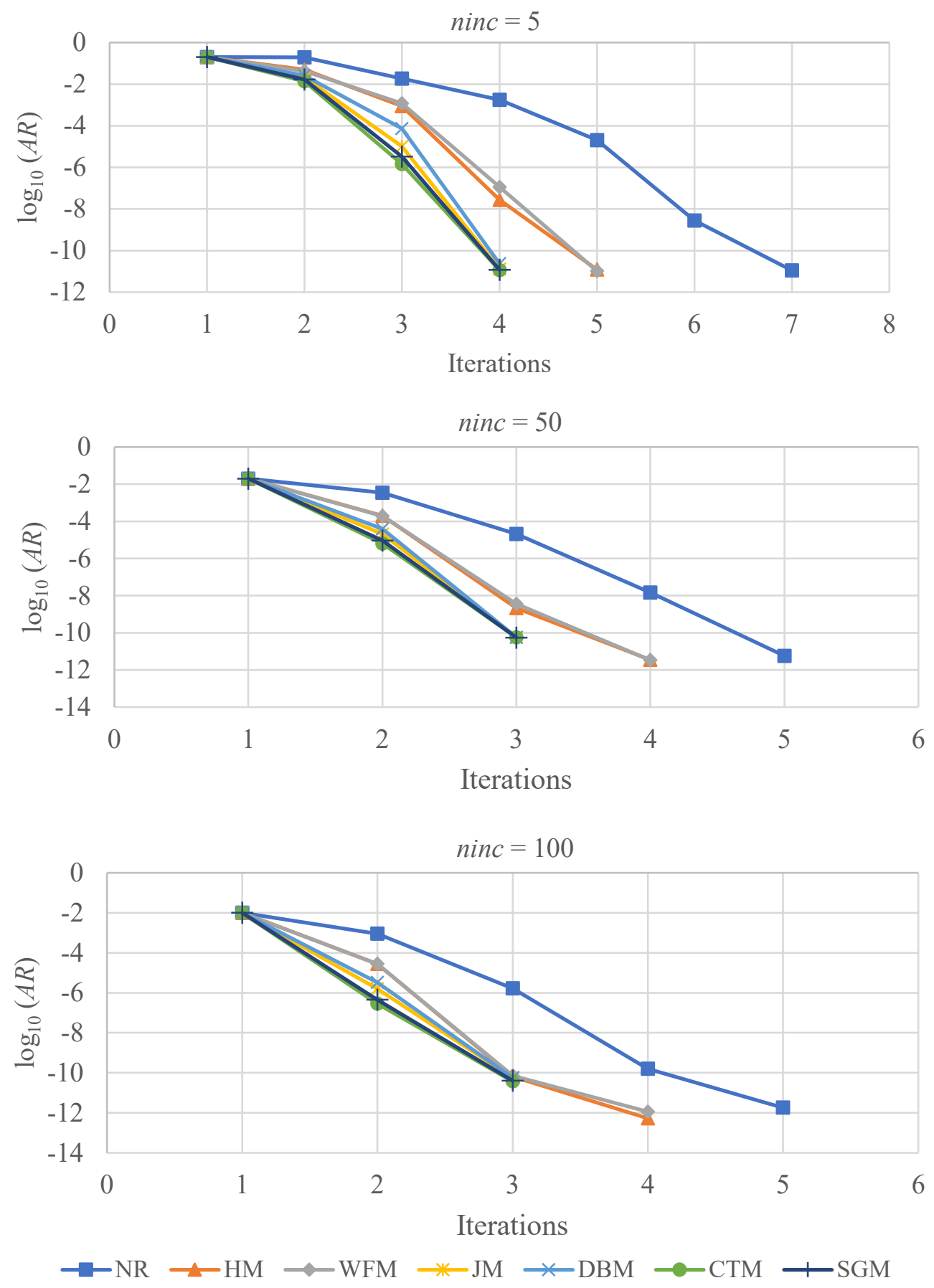

Figure 8: Convergence characteristics of different methods in nonlinear analysis of Schewdeler's dome truss 
To show the variation of the number of iterations in different analysis cases, ninc and the corresponding number of iterations required to trace the equilibrium path is shown in Fig. 9. As can be seen in this figure, the higher order methods require a lower number of iterations in all the load increment size cases. Literally, the main reason is the convergence speed in different methods. As can be observed in Fig. 9, the fifth order methods have a better convergence rate than the fourth order methods and the cubic convergence methods perform better than Newton-Raphson method. As ninc increases, the CTM and SGM show a better performance than the other presented techniques (Fig. 9). It can be concluded that in the high level of fidelity that the applicant requires numerous converged points on the path, the fifth order techniques (CTM and SGM) due to their convergence speed, performs better among the other techniques.

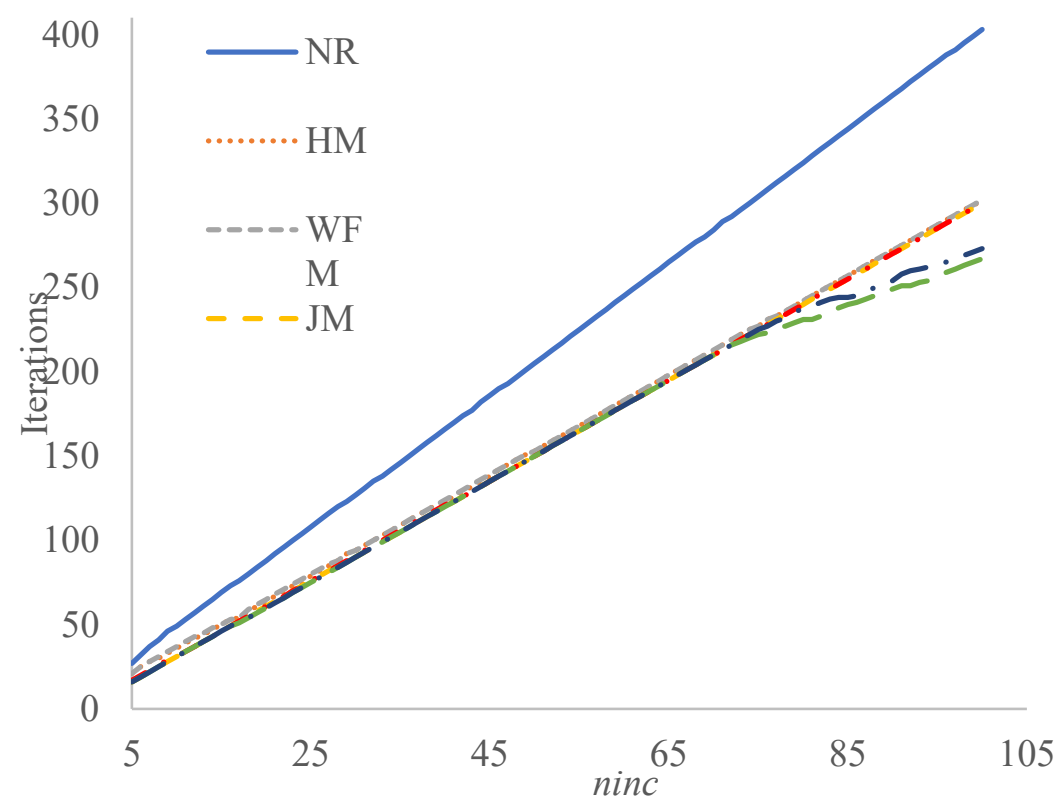

Figure 9: number of iterations vs. ninc curve in nonlinear analysis of Schewdeler's dome truss

Fig. 10 compares the relative percentage reduction (RPR\%) of iterations for higher order methods when compared to the Newton-Raphson method. In this figure, the variation of RPR is plotted for different load increment size cases to compare the behavior of the higher order methods in the different ninc. As can be seen in this figure, HM and WFM have a lower amount of RPR in all the situations than the other higher order methods. These two cubic methods reduce the number of the iterations with respect to the Newton-Raphson method, but as they have lower convergence speed than the other higher order methods, their RPR is lower in all the fidelity levels. In the higher values of ninc (high level of fidelity), the performance of the fourth order and the cubic methods are similar together, but the fifth order techniques (CTM and SGM) show better performance than the others (Fig. 10). It can be concluded that for different level of fidelities the fifth order methods have the highest RPR, but in the high level of fidelity, the fifth order methods because of 
their convergence speed, have a significantly better performance when they are compared to the other higher order methods.

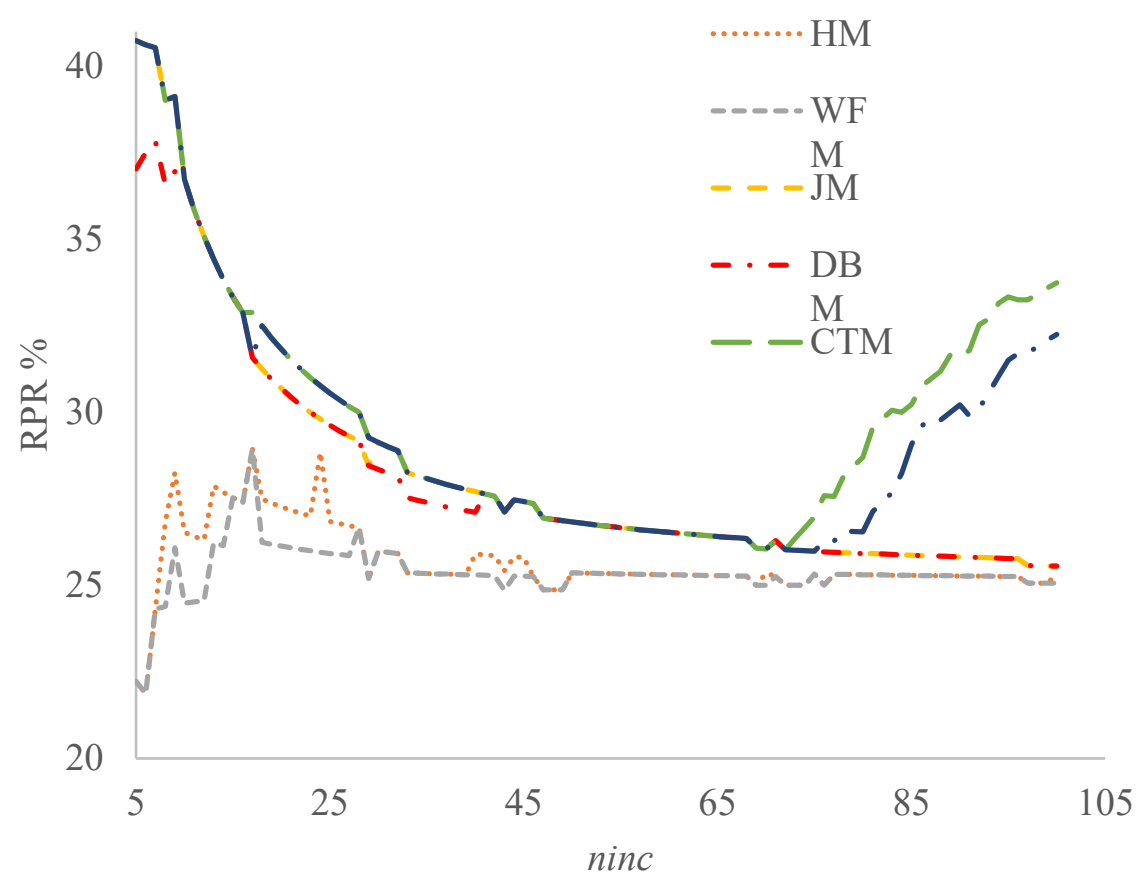

Figure 10: relative percentage reduction (RPR\%) of iterations $v s$. ninc graph in nonlinear analysis of Schewdeler's dome truss for higher order methods

\subsection{Shallow spherical shell}

Fig. 11 shows the shallow spherical shell that its geometry is formed as a cut from a sphere of radius $R$. The structure has a circular base with radius $r$, and it is subjected to a concentrated load at its crown. The structure's support condition is rigid. Penning [Penning (1966)] reported the experimental results for the large displacements of this structure for the thickness of $t=0.05$ in, $r=3.8$ in, $R=19.8$ in, Poisson's ratio of $v=0.3$, and Young's modulus of $E=1.06 e+7 \mathrm{psi}$. The structure is modeled with 176 shell elements. The experimental results of this structure have been used for the verification of the numerical results in Levy et al. [Levy and Spillers (2013); Maghami, Shahabian and Hosseini (2018)].

The represented algorithms are used to analyze the shallow spherical structure. The loaddisplacement curve obtained from the numerical methods is compared to the experimental results in Fig. 12. The maximum relative displacement difference between the numerical results and the experimental results at the same load in Fig. 12 is $2.49 \%$. As it is clear in this figure, the numerical results show a good agreement with the experimental results. The stress contour plots of deformed shape of spherical shell is represented in Fig. 13. Fig. 14 shows the contour plots of deformation in deformed configuration of the spherical shell. 


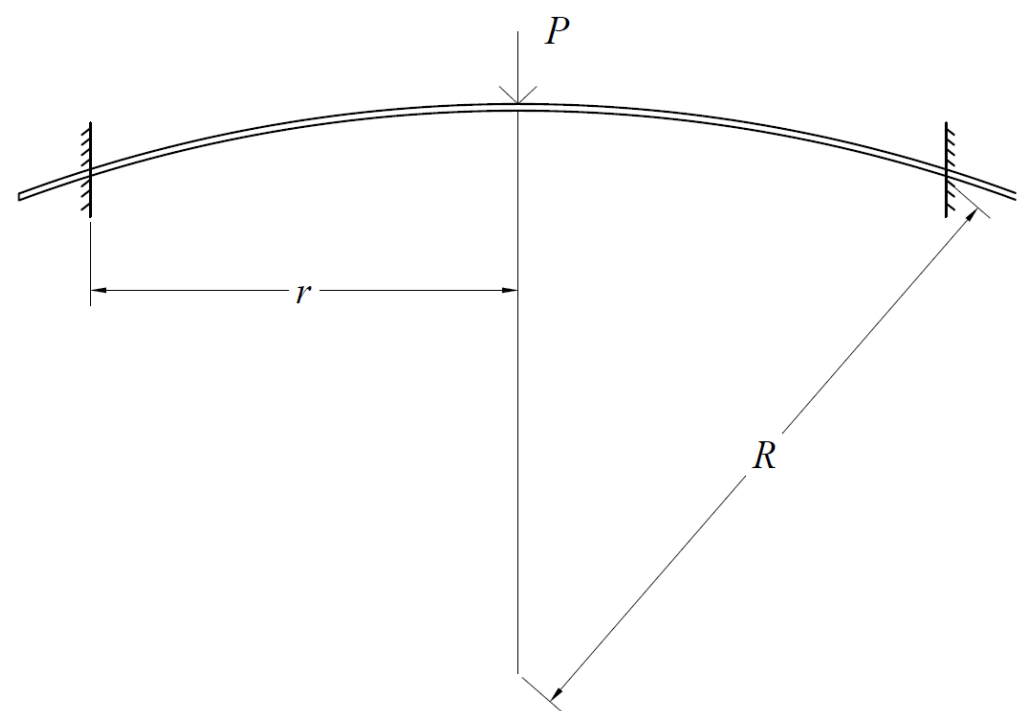

Figure 11: The shallow spherical shell structure

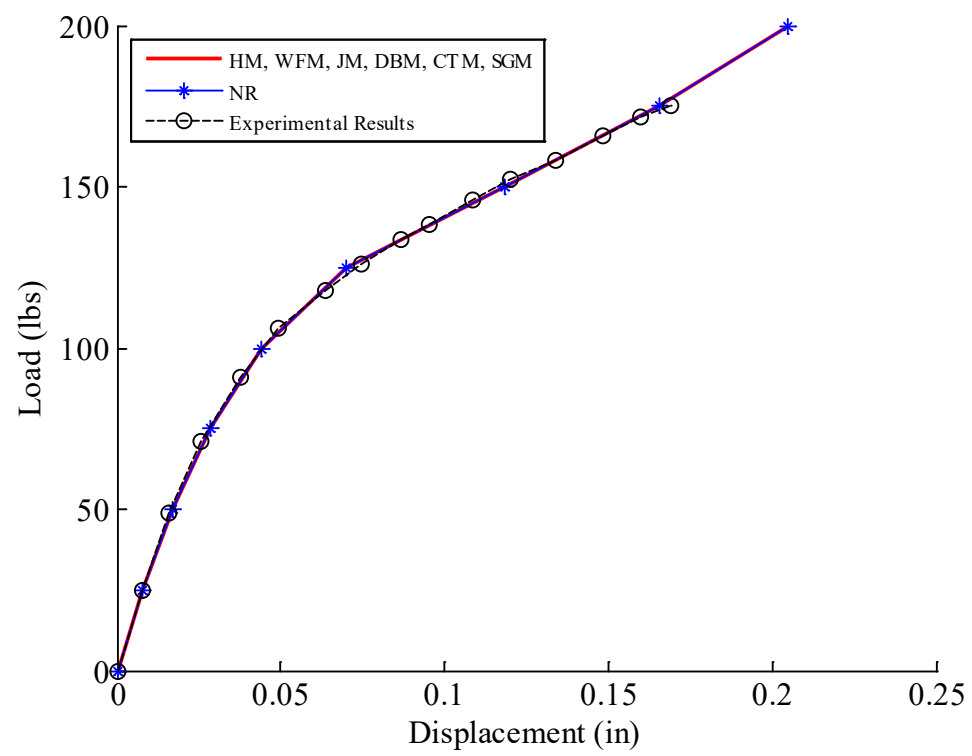

Figure 12: The nonlinear load-displacement curve of shallow spherical shell structure 

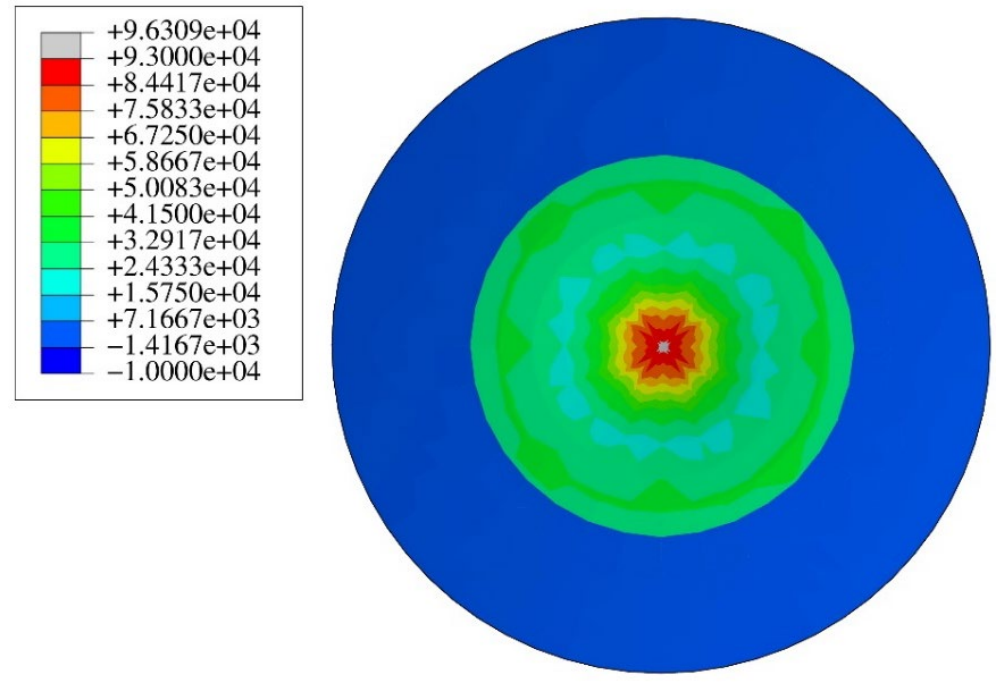

Figure 13: The contour plots of stress in shallow spherical shell structure after deformation
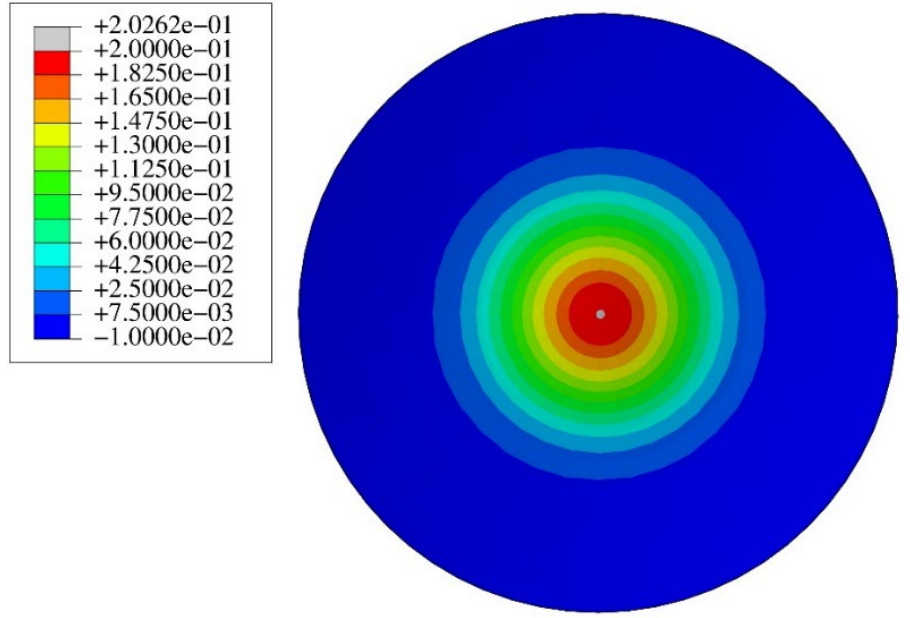

Figure 14: The contour plots of displacements in shallow spherical shell structure after deformation

To compare the computational costs and the sensitivity of the higher order methods to the variation of load increment size, the numerical results related to the higher order methods in 5 cases are provided in Tab. 3. In these five load increment size cases, all the higher order methods require a lower number of iterations to show convergence. It is quite clear that the improvement of fidelity (increase in the number of load increment) leads to an increase in the number of iterations in all the numerical methods. As can be seen in the numerical results of cubic convergence methods, HM requires lower number of iterations and computational cost than the WFM in all the five loading cases. Although in some cases, the required number of iterations of the JM is more than the DBM, the computational cost of JM to perform as a fourth order method is lower than the DBM in all the five situations shown in Tab. 3. By 
comparing the two fifth order methods, it can be seen that the SGM requires a lower computational costs than the CTM in the five cases (Tab. 3). It should also be emphasize that due to the convergence speed of the fifth order methods, the required number of iterations to show convergence in these techniques is lower than the other methods.

Table 3: comparison of different methods in various number of load increments for analysis of shallow spherical shell structure

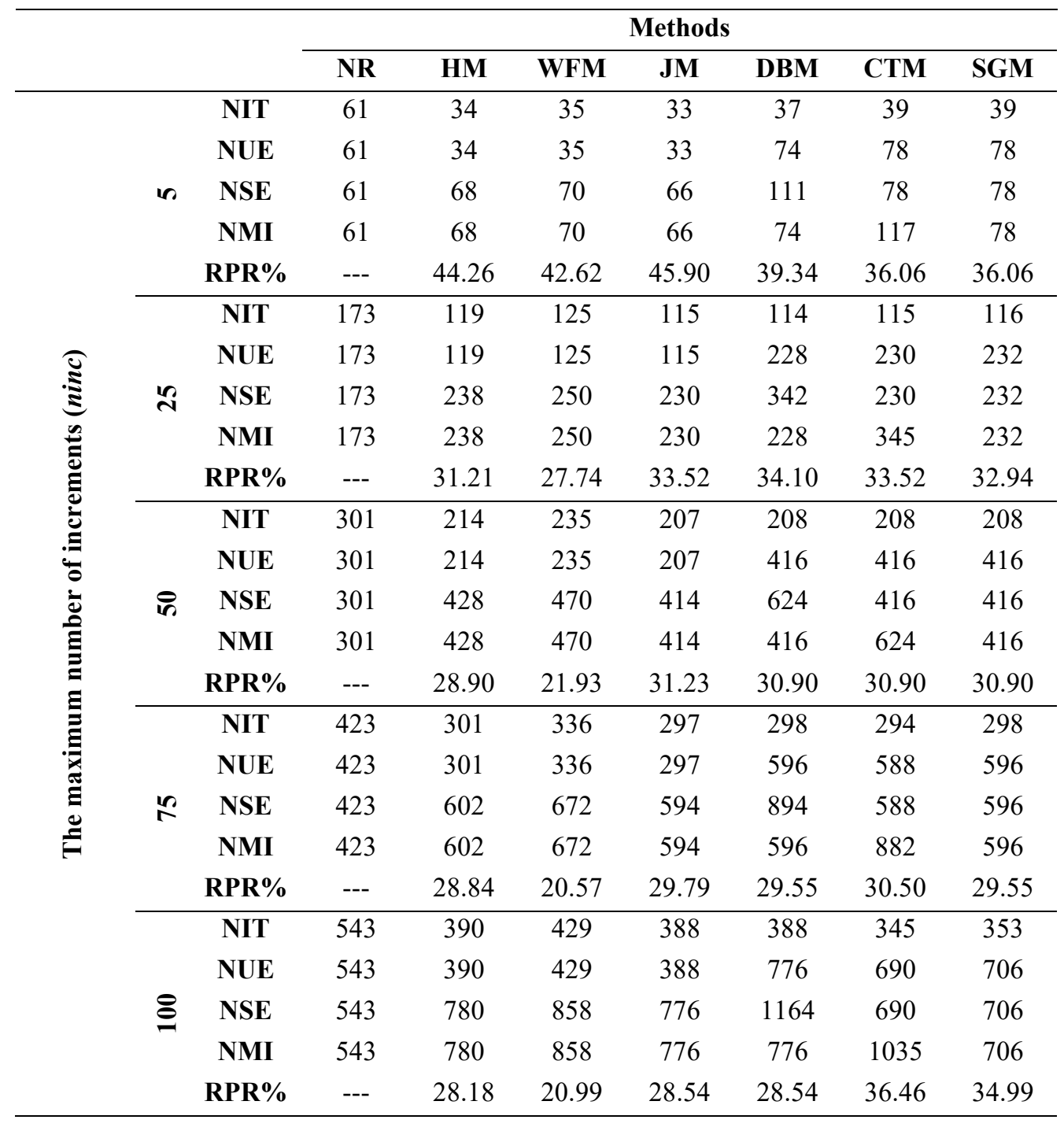



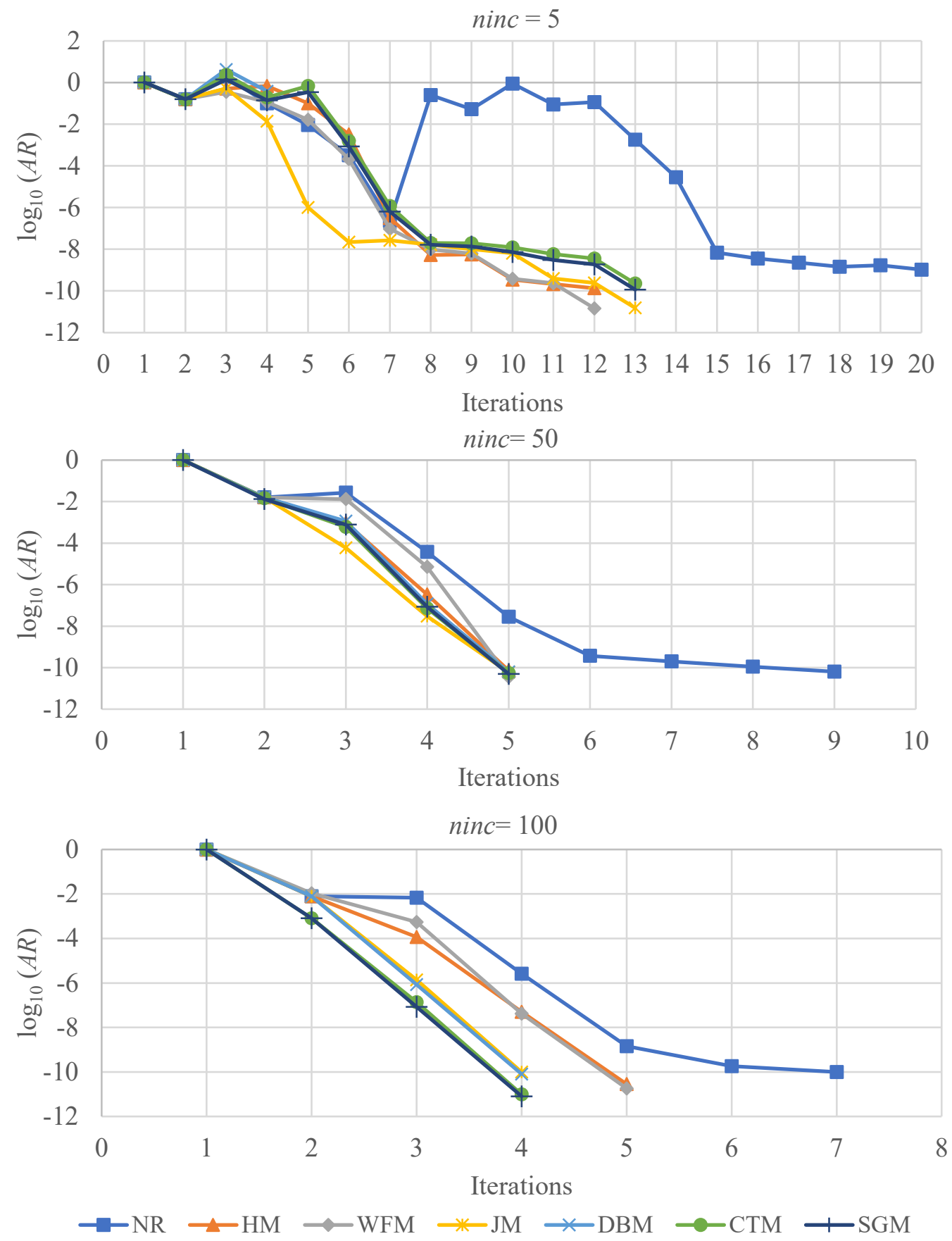

Figure 15: Convergence characteristics of different methods in nonlinear analysis of shallow spherical shell structure 
The variation of the average residual along the iterations are shown in Fig. 15. This figure shows the performance of the higher order methods to reduce the residual in a lower number of iterations when it is compared to the Newton-Raphson method. In fact, the convergence path of numerical methods in Fig. 15 shows the reason that they perform differently to achieve convergence. WFM and HM reduce the average residual in a similar way in the three cases. As mentioned earlier, WFM and HM have the same convergence rate, and their similar behavior is expected. As can be seen in Figure 15, the fourth and the fifth order methods have similar convergence characteristics in the analysis of the shallow shell. It worth recalling that at the third shown case with ninc equal to 100 that represent a high level of fidelity in this example, the convergence path of the fifth order methods (SGM and CTM) is better than that of the fourth order methods (JM and DBM).

Fig. 16 shows the variation of the number of iterations with respect to the variation of ninc. This figure could help to figure out the relation between the fidelity level and the required number of iterations in different methods. Also, Fig. 16 can be utilized to compare the sensitivity of the Newton-Raphson method and the higher order methods. At the lower amount of ninc, the NR method shows sudden variations in the number of iterations that is due to the sensitivity of this method to the size of load increments. As could be seen in Fig. 16, the Newton-Raphson method requires more number of iterations to converge in all the loading cases. As a matter of fact, the other methods have a higher convergence rate that leads to a lower number of iterations comparing to the Newton-Raphson method. The HM has the best performance among the cubic methods and the fifth order techniques (CTM and SGM) show a better performance in a high amount of ninc (Fig. 16). Therefore, in small increment sizes that the applicant require high fidelity level, the fifth order methods have the best efficient performance comparing to the other higher order methods. It must be stressed that the pleasant result of the employed methods in term of the number of iterations is due to the convergence speed.

The variation of RPR for different higher order methods are plotted in Fig. 17. This figure indicate the relation between the fidelity level and RPR for higher order methods. As could be seen in Fig. 17, the numerical results of the higher order methods are obtained in a different values of ninc. In fact, this figure can be used to compare the characteristics of higher order methods in different load increment sizes and fidelity levels. The two third order methods (WFM and HM) have lower convergence speed than the other higher order methods, as a consequence WFM has the least amount of RPR in all the load increment sizes (fidelity levels). The two fourth order methods (JM and DBM) have nearly the same performance in the nonlinear analysis of the shallow spherical shell (Fig. 17). Because of the convergence rate, their performance is better than the third order methods. As the fifth order methods has the best convergence speed among the solution techniques, it has the most RPR in all cases in Fig. 17. 


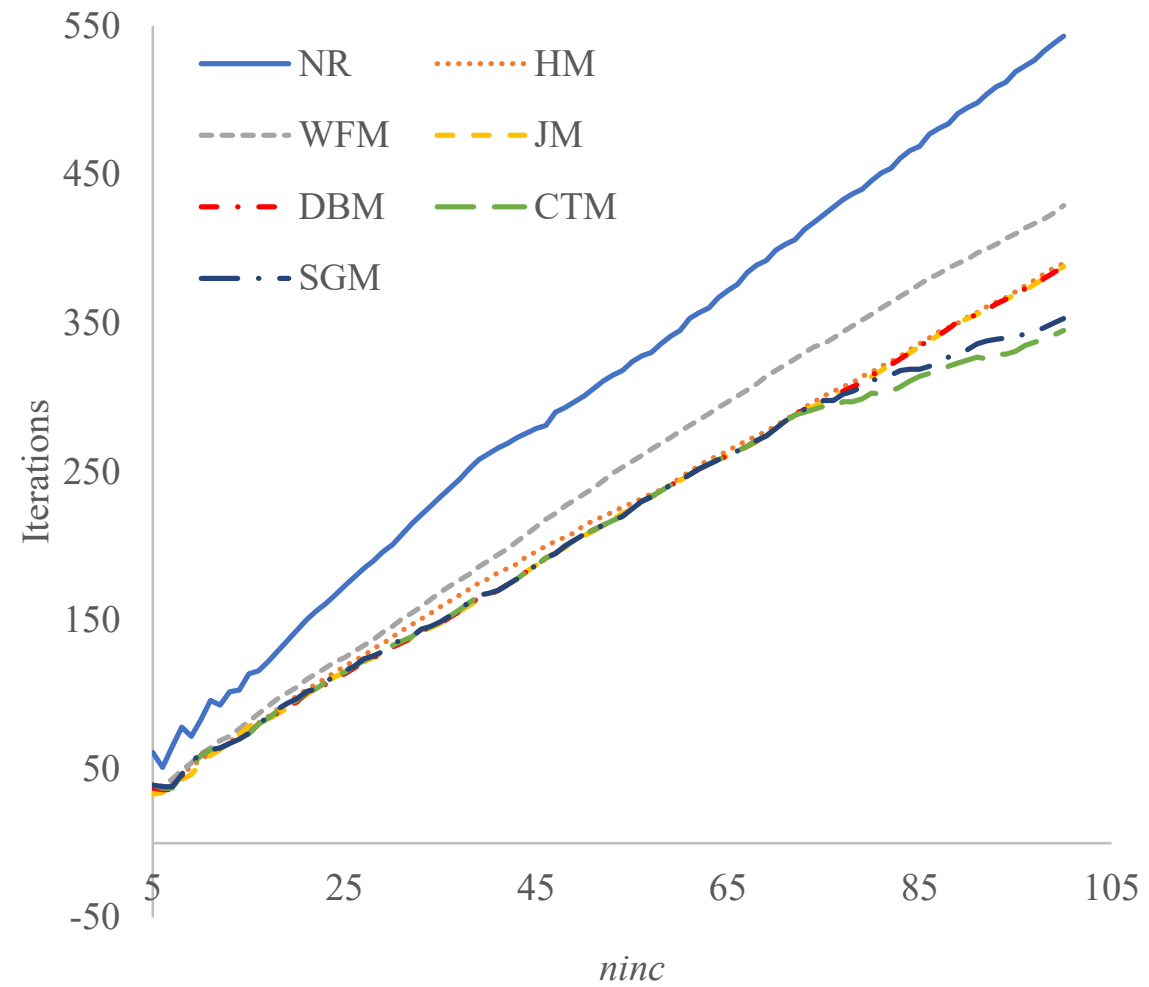

Figure 16: number of iterations $v s$. ninc curve in nonlinear analysis of shallow spherical shell structure

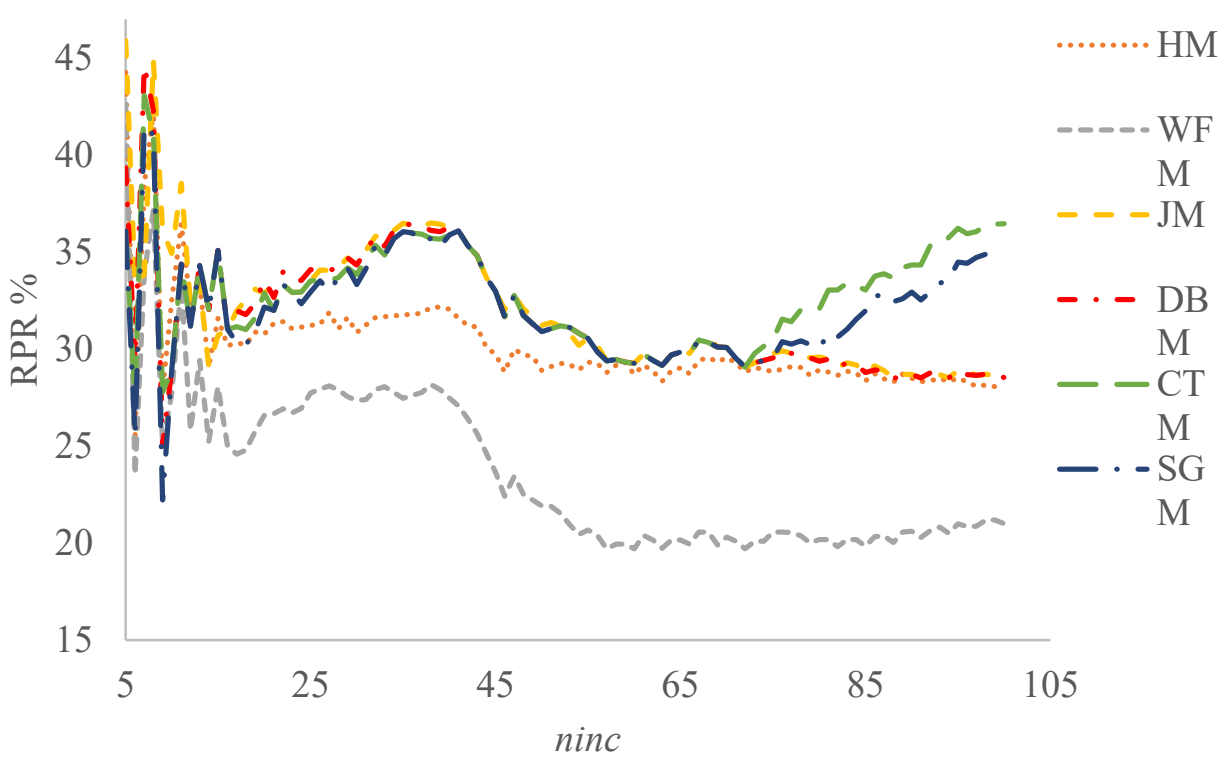

Figure 17: relative percentage reduction (RPR\%) of iterations $v$ s. ninc graph in nonlinear analysis of shallow spherical shell structure for different higher order methods 


\section{Conclusions}

A comparative study on the application of higher order methods in geometrical nonlinear analysis of structures is presented. The convergence characteristics and efficiency of the employed methods are investigated. Six step by step algorithms are presented which are appropriate for the nonlinear analysis using the higher order methods. The higher order methods consist of two cubic (HM and WFM), two fourth order (JM and DBM) and two fifth order (CTM and SGM) techniques. For the comparison of the performance of these algorithms two different structures are analyzed. To show the ability of the six methods, both continuum and discrete problems are chosen as the numerical examples. The first example is a dome truss and the second one is a shell structure. In the second example, the numerical results are compared with the experimental results and the Newton-Raphson's results as well.

The numerical analysis is done by a various load increment size cases to investigate the proficiency of the higher order methods in different fidelity levels. The main results can be outlined as:

- The higher order methods require a lower number of iterations to show convergence than the classical Newton-Raphson method in all load increment size cases (fidelity levels) of the examples.

- As the fifth order methods have the best convergence rate among the other investigated techniques, these methods (CTM and SGM) require the least number of iterations among all the other methods to converge. These techniques have their best performance in small step sizes (high levels of fidelity).

- The convergence characteristics of the presented algorithms reveal that these techniques accelerate the reduction of the residual during the analysis in all step size cases. Indeed, the convergence path of the methods shows that the higher order methods are able to trace a better path to achieve the convergence that is due to their convergence rate.

- As the performance of the cubic methods show, HM has a better performance than WF in both the truss and the shell examples. As a matter of fact, these two techniques has the same order of convergence, but HM is able to converge in lower number of iterations in almost every analysis.

- The performance of JM shows that it requires lower computational cost than the DBM for the analysis of both truss and shell structures in almost every increment load size cases. It is also worth noting that JM has two steps, while DBM works in three steps to achieve fourth order convergence. This is the main reason that the two methods work in different way.

- Among the fifth order methods, SGM requires the least computational cost in all the analysis cases. Actually, this method has the lowest number of iterations among all the solution methods in all fidelity levels.

Acknowledgments: The authors would like to express their gratitude to anonymous reviewers for their in-depth comments that significantly strengthened this article. 


\section{References}

Alamatian, J. (2012): A new formulation for fictitious mass of the dynamic relaxation method with kinetic damping. Computers \& Structures, vol. 90, pp. 42-54.

Arroyo, V.; Cordero, A.; Torregrosa, J. R. (2011): Approximation of artificial satellites' preliminary orbits: the efficiency challenge. Mathematical and Computer Modelling, vol. 54, no. 7-8, pp. 1802-1807.

Brew, J. S.; Brotton, D. M. (1971): Nonlinear structural analysis by dynamic relaxation. International Journal for Numerical Methods in Engineering, vol. 3, no. 4, pp. 463-483

Cai, Y.; Paik, J. K.; Atluri, S. N. (2009): Large deformation analyses of space-frame structures, using explicit tangent stiffness matrices, based on the Reissner variational principle and a von Karman type nonlinear theory in rotated reference frames. Computer Modeling in Engineering \& Sciences, vol. 54, no. 3, pp. 335-368.

Carrera, E. (1994): A study on arc-length-type methods and their operation failures illustrated by a simple model. Computers and structures, vol. 50, no. 2, pp. 217-229.

Cordero, A.; Torregrosa, J. R. (2007): Variants of Newton's method using fifth-order quadrature formulas. Applied Mathematics and Computation, vol. 190, no. 1, pp. 686-698.

Crisfield, M. (1979): A faster modified Newton-Raphson iteration. Computer Methods in Applied Mechanics and Engineering, vol. 20, no. 3, pp. 267-278.

Darvishi, M. T.; Barati, A. (2007a): A fourth-order method from quadrature formulae to solve systems of nonlinear equations. Applied Mathematics and Computation, vol. 188, no. 1, pp. 257-261.

Darvishi, M. T.; Barati, A. (2007b): A third-order Newton-type method to solve systems of nonlinear equations. Applied Mathematics and Computation, vol. 187, no. 2, pp. 630-635.

De Borst, R.; Crisfield, M. A.; Remmers, J. J.; Verhoosel, C. V. (2012): Nonlinear Finite Element Analysis of Solids and Structures. John Wiley \& Sons.

Derakhshandeh, S. Y.; Pourbagher, R. (2016): Application of high-order Newton-like methods to solve power flow equations. IET Generation, Transmission \& Distribution, vol. 10, no. 8, pp. 1853-1859.

Frikha, A.; Wali, M.; Hajlaoui, A.; Dammak, F. (2016): Dynamic response of functionally graded material shells with a discrete double directors shell element. Composite Structures, vol. 154, pp. 385-395.

Ghadiri Rad, M. H.; Shahabian, F.; Hosseini, S. M. (2015a): A meshless local PetrovGalerkin method for nonlinear dynamic analyses of hyper-elastic FG thick hollow cylinder with Rayleigh damping. Acta Mechanica, vol. 226, no. 5, pp. 1497-1513.

Ghadiri Rad, M. H.; Shahabian, F.; Hosseini, S. M. (2015b): Large deformation hyperelastic modeling for nonlinear dynamic analysis of two dimensional functionally graded domains using the Meshless Local Petrov-Galerkin (MLPG) method. Computer Modeling in Engineering \& Sciences, vol. 108, no. 3, pp. 135-157.

Gal, E.; Levy, R. (2006): Geometrically nonlinear analysis of shell structures using a flat triangular shell finite element. Archives of Computational Methods in Engineering, vol. 13, no. 3, pp. 331-388. 
Greco, M.; Gesualdo, F. A.; Venturini, W. S.; Coda, H. B. (2006): Nonlinear positional formulation for space truss analysis. Finite Elements in Analysis and Design, vol. 42, no. 12, pp. 1079-1086.

Green, A. E.; Knops, R. J.; Laws, N. (1968): Large deformations, superposed small deformations and stability of elastic rods. International Journal of Solids and Structures, vol. 4, no. 5, pp. 555-577.

Habibi, A. R.; Bidmeshki, S. (2019): An optimized approach for tracing pre-and postbuckling equilibrium paths of space trusses. International Journal of Structural Stability and Dynamics, vol. 19, no. 04, 1950040.

Hajlaoui, A.; Chebbi, E.; Wali, M.; Dammak, F. (2019) Geometrically nonlinear analysis of FGM shells using solid-shell element with parabolic shear strain distribution. International Journal of Mechanics and Materials in Design, pp. 1-16.

Hajlaoui, A. B.; Triki, E.; Frikha, A.; Wali, M.; Dammak, F. (2017) Nonlinear dynamics analysis of FGM shell structures with a higher order shear strain enhanced solidshell element. Latin American Journal of Solids and Structures, vol. 4, no. 1, pp. 72-91.

Hales, J. D.; Novascone, S. R.; Williamson, R. L.; Gaston, D. R.; Tonks, M. R. (2012): Solving nonlinear solid mechanics problems with the Jacobian-free Newton Krylov method. Computer Modeling in Engineering \& Sciences, vol. 84, no. 2, pp. 123-152.

Han, Z. D.; Rajendran, A. M.; Atluri, S. N. (2005): Meshless Local Petrov-Galerkin (MLPG) approaches for solving nonlinear problems with large deformations and rotations. Computer Modeling in Engineering \& Sciences, vol. 10, no. 1, pp. 1-12.

Homeier, H. H. (2003): A modified Newton method for rootfinding with cubic convergence. Journal of Computational and Applied Mathematics, vol. 157, no. 1, pp. 227-230.

Homeier, H. H. (2004): A modified Newton method with cubic convergence: the multivariate case. Journal of Computational and Applied Mathematics, vol. 169, no. 1 pp. 161-169.

Jarratt, P. (1966): Some fourth order multipoint iterative methods for solving equations. Mathematics of Computation, vol. 20, no. 95, pp. 434-437.

Kelley, C. T. (2003): Solving Nonlinear Equations with Newton's Method. Siam.

Kiran, R.; Khandelwal, K. (2018): On the application of multipoint Root-Solvers for improving global convergence of fracture problems. Engineering Fracture Mechanics, vol. 193, pp. 77-95.

Kiran, R.; Li, L.; Khandelwal, K. (2015): Performance of cubic convergent methods for implementing nonlinear constitutive models. Computers and Structures, vol. 156, pp. 83-100.

Leon, S. E.; Paulino, G. H.; Pereira, A.; Menezes, I. F.; Lages, E. N. (2011): A unified library of nonlinear solution schemes. Applied Mechanics Reviews, vol. 64, no. 4, 040803.

Levy, R.; Gal, E. (2003): Triangular shell element for large rotations analysis. AIAA Journal, vol. 41, no. 12, pp. 2505-2508.

Levy, R.; Spillers, W. R. (2013): Analysis of Geometrically Nonlinear Structures. Springer Science \& Business Media. 
Li, W.; Nguyen-Thanh, N.; Huang, J.; Zhou, K. (2020): Adaptive analysis of crack propagation in thin-shell structures via an isogeometric-meshfree moving least-squares approach. Computer Methods in Applied Mechanics and Engineering, vol. 358, 112613.

Li, W.; Nguyen-Thanh, N.; Zhou, K. (2018): Geometrically nonlinear analysis of thinshell structures based on an isogeometric-meshfree coupling approach. Computer Methods in Applied Mechanics and Engineering, vol. 336, pp. 111-134.

Liu, C. S.; Atluri, S. N. (2008): A novel time integration method for solving a large system of non-linear algebraic equations. Computer Modeling in Engineering \& Sciences, vol. 31, no. 2, pp. 71-83.

Liu, C. S.; Atluri, S. N. (2011): An iterative algorithm for solving a system of nonlinear algebraic equations using the system of ODEs. Computer Modeling in Engineering \& Sciences, vol. 73, no. 4, pp. 395-431.

Liu, C. S.; Yeih, W.; Kuo, C. L.; Atluri, S. N. (2009): A scalar homotopy method for solving an over/under-determined system of non-Linear algebraic equations. Computer Modeling in Engineering \& Sciences, vol. 53, no. 1, pp. 47-71.

Maghami, A.; Shahabian, F.; Hosseini, S. M. (2018): Path following techniques for geometrically nonlinear structures based on Multi-point methods. Computers and Structures, vol. 208, pp. 130-142.

Nguyen, T. N.; Lee, S.; Nguyen-Xuan, H.; Lee, J. (2019): A novel analysis-prediction approach for geometrically nonlinear problems using group method of data handling. Computer Methods in Applied Mechanics and Engineering, vol. 354, pp. 506-526.

Nguyen-Thanh, N.; Li, W.; Zhou, K. (2018): Static and free-vibration analyses of cracks in thin-shell structures based on an isogeometric-meshfree coupling approach. Computational Mechanics, vol. 62, no. 6, pp. 1287-1309.

Nguyen-Thanh, N.; Zhou, K.; Zhuang, X.; Areias, P.; Nguyen-Xuan, H. et al. (2017): Isogeometric analysis of large-deformation thin shells using RHT-splines for multiplepatch coupling. Computer Methods in Applied Mechanics and Engineering, vol. 316, pp. 1157-1178.

Pagani, A.; Carrera, E. (2017): Large-deflection and post-buckling analyses of laminated composite beams by Carrera Unified Formulation. Composite Structures, vol. 170, pp. 40-52.

Pagani, A.; Carrera, E.; Augello, R. (2019): Evaluation of various geometrical nonlinearities in the response of beams and shells. AIAA Journal, vol. 57, no. 8.

Penning, F. A. (1966): Experimental buckling modes of clamped shallow shells under concentrated load. Journal of Applied Mechanics, vol. 33, no. 2, pp. 297-304.

Petkovic, M.; Neta, B.; Petkovic, L.; Dzunic, J. (2012): Multipoint Methods for Solving Nonlinear Equations. Academic Press.

Petkovic, M. S.; Neta, B.; Petkovic, L. D.; Dzunic, J. (2014): Multipoint methods for solving nonlinear equations: a survey. Applied Mathematics and Computation, vol. 226, pp. 635-660.

Rezaiee-Pajand, M.; Naserian, R. (2018): Geometrical nonlinear analysis based on optimization technique. Applied Mathematical Modelling, vol. 53, pp. 32-48. 
Ritto-Correa, M.; Camotim, D. (2008): On the arc-length and other quadratic control methods: Established, less known and new implementation procedures. Computers and Structures, vol. 86, pp. 1353-1368.

Saffari, H.; Maghami, A.; Mansouri, I. (2015): Preconditioned improved bi-conjugate gradient in nonlinear analysis of space trusses. Asian Journal of Civil Engineering, vol. 15, no. 4 , pp. 547-561.

Schillinger, D.; Düster, A.; Rank, E (2012): The hp-d-adaptive finite cell method for geometrically nonlinear problems of solid mechanics. International Journal for Numerical Methods in Engineering, vol. 89, no. 9, pp. 1171-1202.

Sereeter, B.; Vuik, C.; Witteveen, C. (2019): On a comparison of Newton-Raphson solvers for power flow problems. Journal of Computational and Applied Mathematics, vol. 360, pp. 157-169.

Sharma, J. R.; Gupta, P. (2014): An efficient fifth order method for solving systems of nonlinear equations. Computers \& Mathematics with Applications, vol. 67, no. 3, pp. 591-601.

Sharma, J. R.; Sharma, R. (2010): Modified Jarratt method for computing multiple roots. Applied Mathematics and Computation, vol. 217, no. 2, pp. 878-881.

Sotoudehnia, E.; Shahabian, F.; Aftabi-Sani, A. (2019): An iterative method for damage identification of skeletal structures utilizing biconjugate gradient method and reduction of search space. Smart Structures and Systems, vol. 23, no. 1, pp. 45-60.

Torkamani, M. A.; Shieh, J. H. (2011): Higher-order stiffness matrices in nonlinear finite element analysis of plane truss structures. Engineering Structures, vol. 33, no. 12, pp. 3516-3526.

Traub, J. F. (1982): Iterative Methods for the Solution of Equations. American Mathematical Society.

Wang, Y.Z.; Li, F. M. (2015): Nonlinear postbuckling of double-walled carbon nanotubes induced by temperature changes. Applied Physics A, vol. 121, no. 2, pp. 731-738.

Wang, Y.; Li, F. M.; Wang, Y.; Jing, X. (2017): Nonlinear responses and stability analysis of viscoelastic nanoplate resting on elastic matrix under 3:1 internal resonances. International Journal of Mechanical Sciences, vol. 128, pp. 94-104. 